\title{
On Generalized Quantum Stochastic Counting Processes
}

\author{
Bernd Müller ${ }^{\star}$ \\ Institut für Theoretische Physik, Universität Tübingen, Auf der Morgenstelle 14, D-72076 Tübingen, \\ Germany
}

Received: 29 March 1993/in revised form: 13 February 1995

\begin{abstract}
We study counting processes introduced by Davies [11] on general state spaces. The concept of a refinement of a counting process (CP), corresponding to the possibility of distinguishing particles, for instance according to their energy or phase, is introduced, and refinements of general CP's are classified. Then CP's with bounded interaction rate are classified on general state spaces, and sufficient conditions are given in order that the operators characterizing the interaction rate can be formulated in the Schrödinger picture. For CP's with unbounded interaction rates it is shown that analogous to the case of bounded interaction rates there is a family of operators characterizing the interaction rate. Commutation relations for such processes are derived. For constructions of CP's with unbounded interaction rate it is shown that it essentially suffices to solve the semigroup perturbation problem. Finally refinements of these CP's are characterized by "measures" $E \rightarrow J(E)$ on the set of different particles, where each $J(E)$ in an (unbounded) operator.
\end{abstract}

\section{Introduction}

One of the most important problems in quantum optics is the detection of photons $[1,2,24]$ (see [1] for further references). As the corresponding measurements are continuous in time, the usual quantum mechanical formalism, using selfadjoint operators on Fock space, is not applicable and there arises the necessity of a quantum stochastic calculus. Earlier attempts like those of Mandel, Glauber and others $[16,24,25]$ led to unphysical consequences such as the counting probability becoming negative for large time. For a single mode free field Mollow [28] and Scully and Lamb [29] derived another formula free of these problems coinciding with the Mandel formula for small times. But the derivations of this formula are not satisfactory from the point of view of measurement theory as they only consider a single measurement carried out at time $t$. For a detailed critique of the conventional methods see [13]. Davies [10,11] introduced a formalism allowing to treat this

^ e-mail: Bernd Müller: ptim101@mailserv.zdv.uni-tuebingen.de 
problem in a mathematically rigorous way. For some coupling models of photon and detection systems, similar formulae for the fundamental probabilities to those of Davies are derived by a variety of methods [1,2,22,23], however Davies' theory is mathematically full developed and model independent. The ingredients for his theory are a state space $(\mathscr{V}, \mathscr{K}, \tau)$, containing the possible states of the particles, and a measurable space $(X, \Sigma)$. Very often $\mathscr{V}$ is the set of trace class operators on a Hilbert space $\mathscr{H}$ and then $\tau=\mathbb{1} \in \mathscr{B}(\mathscr{H})$. If particles can be distinguished according to some parameter (e.g. energy) $X$ is the set of possible parameter values. If there is no distinction between particles, $X$ consists only of one single point, and we then always choose $X=\{1\}$. The measurement output of a counting experiment performed up to time $t$ has the form,

$$
\left(\left(x_{1}, t_{1}\right), \ldots,\left(x_{n}, t_{n}\right)\right), \quad 0 \leqq t_{1}<t_{2}<\cdots<t_{n}<t,
$$

which means that at time $t_{i}$ a particle with parameter value $x_{i}$ has been detected. If the output is in a set $E$, this induces a change of the state $\rho \rightarrow \mathscr{E}_{t}(E) \rho$ depending on the set $E$. Each operation $\mathscr{E}_{t}(E)$ is a positive linear map on $\mathscr{V}$ and the probability for the event $E$ is given by $\operatorname{tr}\left(\mathscr{E}_{t}(E) \rho\right)$. The set of all possible outcomes is denoted by $X_{t}$ and the event that no particles have been detected is denoted by $\left\{z_{t}\right\}$. The Markov properties (cf. Sect. 2) imply that $S_{t}:=\mathscr{E}_{t}\left(\left\{z_{t}\right\}\right)$ and $T_{t}:=\mathscr{E}_{t}\left(X_{t}\right)$ form semigroups. Davies considered in particular the case of a bounded interaction rate on the state space $\mathscr{V}$ of trace class operators on a Hilbert space. He required that the semigroup $S_{t}$ leaves the set of pure states invariant, in which case $S_{t}$ is called a pure semigroup, and that the probability of detecting one or more particles during the time interval $[0, t)$ gets linearly small in $t$. The main result was that any counting process of this type is determined by the generator $Y$ of a contraction semigroup $B_{t}$ on the Hilbert space $\mathscr{H}$ such that $S_{t} \rho=B_{t} \rho B_{t}^{*}$, and an operator valued measure $A \rightarrow J(A)$ on $(X, \Sigma)$, where each $J(A)$ is a positive operator on $\mathscr{V}$. The operators $J(A)$ describe the instantaneous change of the state induced by the detection of a particle with parameter value in $A . Y$ and $J$ are related by the equation

$$
\operatorname{tr}(J(X)|\psi\rangle\langle\psi|)=-2 \mathbf{R e}\langle\psi, Y \psi\rangle
$$

for all $\psi$ in the domain $D(Y)$ of $Y$, which expresses that the probability of detecting zero or more particles is always one. Moreover $J(X)$ is the (bounded) difference between the generators $Z$ and $W$ of $T_{t}$, resp. $S_{t}$. For applications in quantum optics $[12,13,18,26,27,30]$ one usually neglects the possibility of detecting different kinds of particles, and therefore only considers one operator, for instance $J \rho=a \rho a^{*}$ with the (formal) photon creation and annihilation operators $a^{*}, a$. But this formula induces a series of problems. First of all $J$ is unbounded, which is not only a mathematical complication but is also of physical interest: The main reason for using an operator $J$ for the construction of physically relevant models is that the above classification theorem says that there is no other possibility. For unbounded interaction rates there is no such theorem, moreover there is not even a definition of the term "unbounded interaction rate." Thus we try to get a definition for such processes (5.1) from physical arguments (cf. (6) and Sect. 5) and investigate these objects. The second problem is that the creation and annihilation operators depend on the representation of the canonical commutation relations (CCR) [4]. Moreover, for macroscopic coherent Laser light a detailed analysis [19-21] of Glauber's coherence condition [16] shows that for many important applications the relevant representation of the CCR is not quasi-equivalent to the Fock representation and in 
general has a nontrivial center. In particular the relevant state space is definitely not the set of trace class operators on a Hilbert space. One could restrict the attention to predual spaces of $W^{*}$-algebras, but first there is no mathematical advantage in doing this, and second the use of the general theory shows that the algebraic structure is of no importance for the interpretation and description of counting experiments. Thus in most of the subsequent considerations we are dealing with general state spaces in the sense of the convex state space approach.

The paper is organized as follows. In the next section we introduce the basic concepts and definitions which are all due to Davies. The important class of counting processes with bounded interaction rates is studied in Sect. 4. In Theorems 4.2 and 4.4 we give a complete classification of such processes, but the operators $\Phi(E)\left(=J^{*}(E)\right.$ in the above case $)$ have to be defined in the Heisenberg picture. By introducing semigroups of type $\mathrm{R}$ - which include in particular pure semigroups on $\mathscr{T}_{s a}(\mathscr{H})$ - we can overcome this difficulty and get a direct generalization of Davies' result. Obviously Eq. (1) cannot be formulated on general state spaces. But if $W$ is the generator of the semigroup $S_{t}=\operatorname{Ad} B_{t}$ on $\mathscr{T}_{s a}(\mathscr{H})$ (i.e. $W|\psi\rangle\langle\psi|=| Y \psi\rangle\langle\psi|+| \psi\rangle\langle Y \psi|$ for $\psi \in D(Y)$ ), then one easily verifies that (1) is equivalent to $\tau:=\mathbb{1} \in D\left(W^{*}\right)$ and $W^{*} \tau=-J(X)^{*} \tau$. We always use this form of (1).

In Sect. 5 the first important result is that for unbounded interaction rates formally the same results as in the bounded case are valid, which is the desired justification of the usual way of constructing processes. Moreover, $J(X)$ is the perturbation connecting the two semigroups of the process, and formulae for the computation of probabilities are derived. This shows that the construction of a $\mathrm{CP}$ always involves the solution of the semigroup theoretic perturbation problem. For one point processes, i.e. processes where the space $X$ consists only of one point, Theorem 5.6 shows that it is essentially sufficient to solve this problem in order to construct a CP. But for instance the derivation of Glauber's coherence condition [16] is based on the concept of a detection system that consists of different detectors which are located at different points, so particles can be distinguished according to the locations where they are detected. Other possibilities for a distinction are mass, energy, phase or spin of the particles. In Sect. 3 we see that for each CP $\mathscr{E}_{t}$ there is a corresponding one point $\mathrm{CP}$, the coarsegraining of $\mathscr{E}_{t}$, and therefore the construction of a more complicated $\mathrm{CP}$ can be done in two successive parts: First construct a one point CP and then "refine" it. How refinements may be constructed can be seen in Theorems 3.3 and 5.8. If $J$ is the operator for the one point process, a refinement is characterized by a "measure" $E \rightarrow J(E)$ with $J(X)=J$, where $J(E)$ describes the interaction rate of the detection system for particles of type $E$ (see 5.7 and 5.8 ).

\section{The Basic Concepts}

In the sequel $(\mathscr{V}, \mathscr{K}, \tau)$ is a state space, i.e. $(\mathscr{V}, \mathscr{K})$ is a real ordered Banach space, $\mathscr{K}=\mathscr{V}_{+}$is a normclosed cone with $\mathscr{V}=\mathscr{K}-\mathscr{K}, \tau \in \mathscr{V}^{*}$ is a linear functional satisfying $\left.\tau\right|_{\mathscr{K}}=\|\cdot\|$, and for each $\omega \in \mathscr{V}$ we have $\|\omega\|=\inf \left\{\left\|\omega_{1}\right\|+\left\|\omega_{2}\right\| \mid \omega=\right.$ $\left.\omega_{1}-\omega_{2}, \omega_{l} \in \mathscr{K}\right\}$. This definition can be motivated by the convex state space approach [15], and is a very general description of a physical system. Clearly the predual spaces of a $W^{*}$-algebra or a JBW-algebra are state spaces in this sense $[3,17]$. For $x \in \mathscr{V}^{*}$ we use the duality symbol $\langle\omega, x\rangle:=x(\omega)$. Any $\omega \in \mathscr{V}_{+}$is 
called a state (for reasons explained in [11] we do not require the normalization $\langle\omega, \tau\rangle=\|\omega\|=1)$. A direct consequence of this definition is that a state space is monotone complete, i.e. any monotone bounded sequence of states is converging in norm. Thus if $(X, \Sigma)$ is a measurable space and $\mathscr{E}: \Sigma \rightarrow \mathscr{B}_{+}(\mathscr{V})$ is a positive operator valued set function, then weak and strong $\sigma$-additivity of $\mathscr{E}$ are equivalent. We call such a $\sigma$-additive map a positive operator valued measure (POVM) on $(X, \Sigma)$. If $\mathscr{E}_{n}$ is a sequence of POVM's such that for all $E \in \Sigma$ and $\omega \in \mathscr{V}$ the

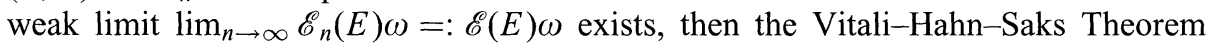
[14] and the above imply that $\mathscr{E}$ again is a POVM. If $\mathscr{E}$ and $\mathscr{F}$ are POVM's on the standard Borel spaces $(X, \Sigma)$ resp. $(Y, \Gamma)$, then there is a unique POVM $\mathscr{G}:=\mathscr{E} \circ \mathscr{F}$ on $(X \times Y, \Sigma \otimes \Gamma)$, called the composition of $\mathscr{E}$ and $\mathscr{F}[10]$ such that

$$
\mathscr{G}(E \times F)=\mathscr{E}(E) \mathscr{F}(F)
$$

for all $E \in \Sigma$ and $F \in \Gamma$. Now we want to describe a measurement of a physical system - characterized by a state $\omega \in \mathscr{V}$ - whose values lie in a measurable space $(X, \Sigma)$. This has to be done by means of an instrument $[10,11]$, i.e. a POVM $\mathscr{E}$ on $(X, \Sigma)$ satisfying $\mathscr{E}(X)^{*} \tau=\tau$. For a normalized $\omega \in \mathscr{V}_{+}$this is connected with the following interpretation:

(i) $\langle\mathscr{E}(E) \omega, \tau\rangle$ is the probability that the result of the measurement is in the set $E \in \Sigma$.

(ii) $\mathscr{E}(E) \omega$ is the state after the measurement conditional upon the results in $E$.

Then a repeated measurement can be described by the composition of the corresponding instruments (cf. Eq. (2)). Obviously any counting experiment, performed from time 0 up to time $t$ generates outcomes of the form

$$
\left(\left(x_{1}, t_{1}\right), \ldots,\left(x_{n}, t_{n}\right)\right), \quad 0 \leqq t_{1}<t_{2}<\cdots<t_{n}<t,
$$

where the $t_{l}$ are the times at which particles are detected, and the $x_{i}$ are elements of a measurable space $(X, \Sigma)$ describing the type of particle. As we have mentioned above, $X$ could be the set of possible energy values of a particle or $X$ could characterize the location of a system. We denote the event that no particle is detected during this time interval by $z_{t}$. The collection of all events where exactly $n$ particles are detected in $[0, t)$ is denoted by $A_{n}^{t}$, thus

$$
A_{n}^{t}:=\left\{\left(\left(x_{1}, t_{1}\right), \ldots,\left(x_{n}, t_{n}\right)\right) \mid 0 \leqq t_{1}<\cdots<t_{n}<t, x_{i} \in X\right\}, \quad A_{0}^{t}:=\left\{z_{t}\right\} .
$$

If we do not distinguish between different kinds of particles, i.e. $X=\{1\}$, we identify the tuples $\left(\left(1, t_{1}\right), \ldots,\left(1, t_{n}\right)\right)$ and $\left(t_{1}, \ldots, t_{n}\right)$, and use the abbreviations

$$
C_{n}^{t}:=\left\{\left(t_{1}, \ldots t_{n}\right) \mid 0 \leqq t_{1}<\cdots<t_{n}<t\right\}, \quad C_{0}^{t}:=\left\{z_{t}\right\} .
$$

Let us define

$$
X_{t}:=\bigcup_{n \in \mathbb{N}} A_{n}^{t}
$$

and canonically identify $\bigcup_{n \geq 0} C_{n}^{t}$ with $\{1\}_{t}$. In principle we could modify the condition in (3) to $0 \leqq t_{1} \leqq \cdots \leqq t_{n} \leqq t$, but it turns out that all relevant measures are absolutely continuous with respect to the Lebesgue measure, so the probability of simultaneously detecting two or more particles is always zero (as one would expect for physical situations). We note that $X_{t}$ and all $A_{n}^{t}$ are standard Borel spaces whenever $X$ is. Now, because of the continuous variability of such an experiment in 
time, it cannot be described by only one instrument on the space $X_{t}$ (for a fixed $t$ ), but by a family $\mathscr{E}_{t}$ of instruments on the spaces $X_{t}$, where each $\mathscr{E}_{t}$ describes the measurement up to the time $t$. Thus we define

2.1. Definition. Let $(X, \Sigma)$ be a measurable space, $(\mathscr{V}, \mathscr{K}, \tau)$ a state space. $A$ family $\left(\mathscr{E}_{t}\right)_{t \geqq 0}$ of instruments on $X_{t}$ is called a counting process $(C P)$ with measurable space $(X, \Sigma)$, if

(i) $\|\cdot\|-\lim _{t \rightarrow 0} \mathscr{E}_{t}\left(X_{t}\right) \omega=\omega$ for all $\omega \in \mathscr{\psi}$, and

(ii) $\mathscr{E}_{t}(F) \mathscr{E}_{s}(E)=\mathscr{E}_{t+s}(\lambda(F \times E))$ for all measurable $E \subseteq X_{s}, F \subseteq X_{t}$,

where $\lambda: X_{t} \times X_{s} \rightarrow X_{t+s}$ is defined by

$$
\begin{aligned}
& \left(\left(\left(x_{1}, t_{1}\right), \ldots,\left(x_{n}, t_{n}\right)\right),\left(\left(y_{1}, s_{1}\right), \ldots,\left(y_{m}, s_{m}\right)\right)\right) \\
& \quad \rightarrow\left(\left(y_{1}, s_{1}\right), \ldots,\left(y_{m}, s_{m}\right),\left(x_{1}, t_{1}+s\right), \ldots,\left(x_{n}, t_{n}+s\right)\right) .
\end{aligned}
$$

In most cases $(X, \Sigma)$ is understood and we thus will only speak of a CP $\mathscr{E}_{t}$ without explicitly mentioning its measurable space. To be correct we should give $\lambda$ a pair of indices $t, s$, but we hope that in the sequel it is always clear which spaces are connected by $\lambda$. Equation 2.1(ii) is similar to the Chapman-Kolmogorov equations for Markov processes in classical probability theory and it expresses that the process has no memory and is homogeneous in time. A direct consequence of 2.1(ii) is that the operations

$$
S_{t}:=\mathscr{E}_{t}\left(\left\{z_{t}\right\}\right) \text { and } T_{t}:=\mathscr{E}_{t}\left(X_{t}\right)
$$

form semigroups; moreover $T_{t}$ is a $C_{0}$-semigroup. We always denote by $Z$ the generator of $T_{t}$ and by $W$ that of $S_{t}$ (if $S_{t}$ is a $C_{0}$-semigroup). For properties of semigroups we refer to $[3,9]$. The above definition allows to calculate the probability for any physical event. For instance the probability $P_{t}(n)$ for detecting exactly $n$ particles during the time interval $[0, t)$ is given by

$$
P_{t}(n)=\left\langle\mathscr{E}_{t}\left(A_{n}^{t}\right) \omega, \tau\right\rangle
$$

for a normalized $\omega \in \psi_{+}$. Thus the average number $\langle N\rangle_{\text {, of }}$ particles detected in this period is

$$
\langle N\rangle_{t}=\sum_{n=0}^{\infty} n P_{t}(n)=\sum_{n=0}^{\infty} n\left\langle\mathscr{O}_{t}\left(A_{n}^{t}\right) \omega, \tau\right\rangle
$$

Moreover the average time $\left\langle t_{a}\right\rangle$ at which the first particle is detected is given by

$$
\left\langle t_{a}\right\rangle=\left.\int_{0}^{\infty} t \frac{d}{d s}\left\langle\left(T_{s}-S_{s}\right) S_{t}(\omega), \tau\right\rangle\right|_{s=0} d t
$$

if $s \rightarrow\left\langle\left(T_{s}-S_{\varsigma}\right) S_{t}(\omega, \tau\rangle\right.$ is differentiable for all $t$. One can see this using the fact that the probability for the first particle being detected in $[t, t+\Delta t)$ is given by $\left\langle\mathscr{E}_{\Delta t}\left(X_{\Delta t} \backslash\left\{z_{\Delta t}\right\}\right) S_{t} \omega, \tau\right\rangle$. We mention this because the differentiability condition implies for physically interesting states $\omega$ that $\|\left(T_{s}-S_{s}\right)(\omega \| \leqq K s$ for small $s$, an inequality which we will use in the two final sections (cf. Eqs. (10) and (29)). Let us now present some further developments of Davies' formalism. 


\section{Coarsegrainings and Refinements}

Physically one may be interested in the connection between the probability distribution in certain channels - represented by the space $(X, \Sigma)$ - and the distribution of the whole system. Mathematically this corresponds to a coarsening procedure that combines all the points of the space $X$ in a single point, e.g. $\{1\}$. We identify the space $\{1\}_{t}$ with $\bigcup_{n \in \mathbb{N}} C_{n}^{t}$ and introduce the "coarsegraining" map

$$
\pi: X_{t} \rightarrow\{1\}_{t}, \quad\left(\left(x_{1}, t_{1}\right), \ldots,\left(x_{n}, t_{n}\right)\right) \rightarrow\left(t_{1}, \ldots, t_{n}\right) .
$$

Obviously $\pi$ is measurable and describes the loss of information about the channels. One now easily verifies the following statement.

3.1. Proposition. Let $(\mathscr{V}, \mathscr{K}, \tau)$ be a state space and $\mathscr{E}_{t}$ a $C P$ with measurable space $(X, \Sigma)$. Then the definition

$$
\widetilde{\mathscr{E}}_{t}(E):=\mathscr{E}_{t}\left(\pi^{-1}(E)\right)
$$

for each measurable $E \subseteq\{1\}_{\text {t }}$ yields a one point $C P$.

We call the resulting CP $\widetilde{\mathscr{E}}_{t}$ the one point coarsegraining of $\mathscr{E}_{t}$. For constructions of CP's the inverse way is important. Before attacking this problem we have a look at the operations determining the process. In the sequel we will often have to deal with sets of the form

$$
A=\left(E_{1} \times\left[a_{1}, b_{1}\right)\right) \times \cdots \times\left(E_{n} \times\left[a_{n}, b_{n}\right)\right),
$$

where $E_{l} \in \Sigma$ and $0 \leqq a_{1}<b_{1}<a_{2}<b_{2}<\cdots<b_{n}<t$. Obviously $A$ is a measurable set in $A_{n}^{t}$. We call a finite union of sets of this form a standard set. The standard sets are closed under the formation of finite intersections and generate the $\sigma$-algebra on $A_{n}^{t}$.

3.2. Lemma. Let $\mathscr{E}_{t}^{1}, \mathscr{E}_{t}^{2}$ be CP's with measurable space $(X, \Sigma)$ fulfilling

(i) $S_{t}^{1}=S_{t}^{2}=: S_{t}$ and

(ii) $\mathscr{E}_{t}^{1}(E \times[0, t))=\mathscr{E}_{t}^{2}(E \times[0, t))$ for all $E \in \Sigma$ and $t>0$,

then $\mathscr{E}_{t}^{1}=\mathscr{E}_{t}^{2}$.

Proof. It is sufficient to verify $\mathscr{E}_{t}^{1}(A)=\mathscr{E}_{t}^{2}(A)$ for $A$ as in Eq. (7). But then we have

$$
\mathscr{E}_{t}^{1}(A)=S_{t-b_{n}} \mathscr{E}_{b_{n}-a_{n}}^{1}\left(E_{n} \times\left[0, b_{n}-a_{n}\right)\right) \cdots \mathscr{E}_{b_{1}-a_{1}}^{1}\left(E_{1} \times\left[0, b_{1}-a_{1}\right)\right) S_{a_{1}}=\mathscr{E}_{t}^{2}(A)
$$

by the Markov property of the CP's.

Now we return to the problem of constructing a "refinement" of a CP. By the above it is enough to give the process on $A_{0}^{t}$ and $A_{1}^{t}$. For $E \in \Sigma$ and $0<a<b<t$ one necessarily has:

$$
\mathscr{E}_{t}(E \times[a, b))=S_{t-b} \mathscr{E}_{b-a}(E \times[0, b-a)) S_{a} .
$$

The subsequent theorem shows that this condition is essentially sufficient. 
3.3. Theorem. Let $\widetilde{\mathscr{E}}_{t}$ be a one point $C P,(X, \Sigma)$ a standard Borel space, and for each $t>0$ let $G_{t}$ be a POVM on $A_{1}^{t}$ satisfying

(i) $G_{t}(X \times[0, t))=\widetilde{\mathscr{E}}_{t}([0, t))$, and

(ii) $G_{t}(E \times[a, b))=S_{t-b} G_{b-a}(E \times[0, b-a)) S_{a}$

for $E \in \Sigma$ and $0<a<b<t$. Then there is a unique $C P \mathscr{E}_{t}$ with measurable space $X$ such that $\mathscr{E}_{t}(A)=G_{t}(A)$ for measurable $A \subseteq X \times[0, t)$, and $\mathscr{E}_{t}\left(\left\{z_{t}\right\}\right)=$ $\widetilde{\mathscr{E}}_{t}\left(\left\{z_{t}\right\}\right)=S_{t}$. Moreover $\widetilde{\mathscr{E}}_{t}$ is the one point coarsegraining of $\mathscr{E}_{t}$.

Proof. The uniqueness is obvious from Lemma 3.2, and the last statement is a direct consequence of (i) and (ii). The rest is technically a bit complicated and we thus first try to sketch the idea. There is a canonical way (cf. Eq. (8) below) of defining $\mathscr{E}_{t}$ on standard sets. However it is not clear if this procedure is well defined and if the map $E \rightarrow \mathscr{E}_{t}(E)$ may be extended $\sigma$-additively. Thus we subdivide the interval $[0, t)$ in $2^{n}$ disjoint intervals $I_{k}^{n}:=\left[\frac{k-1}{2^{n}} t, \frac{k}{2^{n}} t\right)$ for $k=1, \ldots, 2^{n}$ and restrict our attention to the set

$$
\Lambda_{n}^{l}:=\left\{x=\left(\left(x_{1}, t_{1}\right), \ldots,\left(x_{l}, t_{l}\right)\right) \in A_{l}^{t} \mid x_{l} \in X,\right.
$$

for each $k$ there is at most one $\left.t_{i} \in I_{k}^{n}\right\}$.

We say " $x$ has an entry in $I_{k}^{n}$ " iff there is a $t_{i}$ with $t_{l} \in I_{k}^{n}$. On the set $\Lambda_{n}^{l}$ the canonical definition leads to a well defined $\sigma$-additive map, and by use of a limiting procedure we receive a measure satisfying the required conditions. The details are covered by the following lemma.

3.4. Lemma. We use the above notation. Let $S_{t}$ be a positive contractive semigroup, and $G_{t}$ a POVM on $X \times[0, t)$ that fulfills 3.3(ii). Then for fixed there is a sequence $\left(\mu_{n}^{l}\right)_{n \in \mathbb{N}}$ of POVM's on $C_{l}^{t}$ such that

(i) $\mu_{n+1}^{l}(E)=\mu_{n}^{l}(E)$ for measurable $E \subseteq \Lambda_{n}^{l}$,

(ii) $\mu_{n}^{l}\left(C_{l}^{t}\right)=\mu_{n}^{l}\left(\Lambda_{n}^{l}\right)$,

(iii) $\mu_{n+1}^{l}(E) \geqq \mu_{n}^{l}(E)$ for measurable $E \subseteq C_{l}^{t}$.

For $E=\left(E_{1} \times\left[a_{1}, b_{1}\right)\right) \times \cdots \times\left(E_{l} \times\left[a_{l}, b_{l}\right)\right) \subseteq A_{l}^{t}$ there is an $n_{0} \in \mathbb{N}$ such that for $n \geqq n_{0}$,

$$
\mu_{n}^{l}(E)=S_{t-t_{l}} G_{t_{l}-s_{l}}\left(E_{l} \times\left[0, t_{l}-s_{l}\right)\right) \cdots G_{t_{1}-s_{1}}\left(E_{1} \times\left[0, t_{1}-s_{1}\right)\right) S_{s_{1}} .
$$

Proof. We say a map $\varphi:\left\{1, \ldots, 2^{n}\right\} \rightarrow\{0,1\}$ is in $Q_{n}^{l}$, iff card $\varphi^{-1}(\{1\})=l$. For each $\varphi \in Q_{n}^{l}$ we define $P_{\varphi}:=\left\{\psi \in Q_{n+1}^{l} \mid\right.$ for each $k \in \varphi^{-1}(\{1\})$ there is one and only one $l \in\{2 k-1,2 k\}$ with $\psi(l)=1\}$. Obviously the $P_{\varphi}$ are pairwise disjoint. Finally let $C_{0}^{n}:=\left\{z_{t / 2^{n}}\right\}, C_{1}^{n}:=X \times\left[0, t / 2^{n}\right)$, and for each $\varphi \in Q_{n}^{l}$ let $A_{\varphi}:=\lambda\left(C_{\varphi\left(2^{n}\right)}^{n} \times \cdots \times \lambda\left(C_{\varphi(2)}^{n} \times C_{\varphi(1)}^{n}\right) \cdots\right)$. Thus an $x \in A_{\varphi}$ has an entry in $I_{k}^{n}$ iff $\varphi(k)=1$. If $X=\{1\}$ we use $B_{\varphi}$ instead of $A_{\varphi}$ and $\widetilde{\Lambda}_{n}^{l}$ instead of $\Lambda_{n}^{l}$. Now the following statements are easy to verify:

$$
\begin{gathered}
\Lambda_{n}^{l}=\bigcup_{\varphi \in Q_{n}^{l}} A_{\varphi}, \quad A_{\varphi}=\bigcup_{\psi \in P_{\varphi}} A_{\psi}, \\
\Lambda_{n}^{l} \uparrow A_{l}^{t} \text {, i.e. } \Lambda_{n}^{l} \subseteq \Lambda_{n+1}^{l} \quad \text { and } \bigcup_{n \in \mathbb{N}} \Lambda_{n}^{l}=A_{l}^{t} .
\end{gathered}
$$


The $\sigma$-algebra on $A_{\varphi}$ is generated by sets of the form $E=\lambda\left(E_{2^{n}} \times \cdots \times E_{1}\right)$ with measurable sets $E_{i} \subseteq C_{\varphi(l)}^{n}$. For these sets we define

$$
\widetilde{\mu}_{\varphi}(E)=\widetilde{G}_{t / 2^{n}}\left(E_{2^{n}}\right) \cdots \widetilde{G}_{t / 2^{n}}\left(E_{1}\right),
$$

where $\widetilde{G}_{r}\left(E_{i}\right)=G_{r}\left(E_{l}\right)$ for $E_{l} \subseteq C_{1}^{n}$ and $\widetilde{G}_{r}\left(\left\{z_{r}\right\}\right)=S_{r}$. Then using the fact that $\prod_{l=1}^{2^{n}} C_{\varphi(l)}$ is Borel isomorphic to $A_{\varphi}$ it follows by (2) that $E \rightarrow \widetilde{\mu}_{\varphi}(E)$ can be extended to a POVM on $A_{\varphi}$. Now it is a technical but straightforward calculation that for measurable $E \subseteq A_{\varphi}$ we have:

$$
\widetilde{\mu}_{\varphi}(E)=\sum_{\psi \in P_{\varphi}} \widetilde{\mu}_{\psi}\left(E \cap A_{\psi}\right) .
$$

Thus the definition $\mu_{n}^{l}(E):=\sum_{\varphi \in Q_{n}^{l}} \widetilde{\mu}_{\varphi}\left(E \cap A_{\varphi}\right)$ for measurable $E \subseteq A_{l}^{t}$ generates a POVM on $A_{l}^{t}$ that satisfies 3.4(i),3.4(ii) and therefore 3.4(iii). Let $E=\left(E_{1} \times\right.$ $\left.\left[a_{1}, b_{1}\right)\right) \times \cdots \times\left(E_{l} \times\left[a_{l}, b_{l}\right)\right)$ be a standard set with $0 \leqq a_{1}<b_{1}<\cdots<b_{l}<$ $t$, and let $r>0$ be the minimum distance between two successive parts of this inequality, then a direct calculation shows for $\frac{t}{2^{n}}<\frac{r}{4}$ that $E \subseteq \Lambda_{n}^{l}$ and that $\mu_{n}^{l}(E)$ fulfills (8).

Proof of Theorem. By definition of $\mu_{n}^{l}$ and using Theorem 3.3(i) one calculates $\mu_{n}^{l}\left(A_{\varphi}\right)=\widetilde{\mathscr{E}}_{t}\left(B_{\varphi}\right)$, thus

$$
\mu_{n}^{l}\left(A_{l}^{t}\right)=\sum_{\varphi \in Q_{n}^{l}} \mu_{n}^{l}\left(A_{\varphi}\right)=\widetilde{\mathscr{E}}_{t}\left(\bigcup_{\varphi \in Q_{n}^{l}} B_{\varphi}\right)=\widetilde{\mathscr{E}}_{t}\left(\widetilde{\Lambda}_{n}^{l}\right) \leqq \widetilde{\mathscr{E}}_{t}\left(C_{l}^{t}\right) .
$$

Monotonicity and boundedness of this sequence imply that for each measurable $E \subseteq C_{l}^{t}$ and $\omega \in \mathscr{V}_{+}$(and therefore all $\left.\omega \in \mathscr{V}\right)$

$$
\|\cdot\| \lim _{n \rightarrow \infty} \mu_{n}^{l}(E) \omega:=\mathscr{E}_{t} l(E) \omega
$$

exists. As a limit of a sequence of POVM's $\mathscr{E}_{t}$ is a POVM on $A_{l}^{t}$, and for each $\omega \in \mathscr{V}$ we have

$$
\mathscr{\mathscr { E }}_{t}^{l}\left(A_{l}^{t}\right) \omega=\lim _{n \rightarrow \infty} \mu_{n}^{l}\left(A_{l}^{t}\right) \omega=\lim _{n \rightarrow \infty} \widetilde{\mathscr{E}}_{t}\left(\widetilde{\Lambda}_{n}^{l}\right) \omega=\widetilde{\mathscr{E}}_{t}\left(\bigcup_{n \in \mathbb{N}} \widetilde{\Lambda}_{n}^{l}\right) \omega=\widetilde{\mathscr{E}}_{t}\left(C_{l}^{t}\right) \omega
$$

Now we define $\mathscr{E}_{t}(E) \omega:=\sum_{l} \mathscr{E}_{t}\left(E \cap A_{l}^{t}\right) \omega$. Equation (9) implies that the sum is converging, thus $\mathscr{E}_{t}$ is a POVM on $X_{t}$ satisfying

$$
\mathscr{E}_{t}\left(X_{t}\right) \omega=\sum_{n=0}^{\infty} \mathscr{E}_{t} l\left(A_{l}^{t}\right) \omega=\sum_{n=0}^{\infty} \widetilde{\mathscr{E}}_{t}\left(C_{l}^{t}\right) \omega=T_{t} \omega
$$

Therefore it remains to show 2.1(ii). But as it suffices to verify the Markov properties on standard sets, we can use Eq. (8) and the definition of $\mathscr{E}_{t}$ in order to get the desired result.

We call the CP $\mathscr{E}_{t}$ constructed here a refinement of the one point process $\widetilde{\mathscr{E}}_{t}$. The above theorem shows that there is a one-to-one correspondence between the refinements of a given one point CP $\widetilde{E}_{t}$, and the POVM's $G_{t}$ satisfying 3.3(ii). Therefore it allows us to concentrate on one point processes when constructing CP's (cf. Sect. 5). 


\section{Counting Processes with Bounded Interaction Rate}

Here we study a special class of CP's introduced by Davies [11]. A CP $\mathscr{E}_{t}$ is said to have a bounded interaction rate (IR), if there is a $K>0$ such that

$$
\left\|\mathscr{E}_{t}\left(X_{t} \backslash\left\{z_{t}\right\}\right)\right\|=\left\|T_{t}-S_{t}\right\| \leqq K t
$$

for all $t \in \mathbb{R}$. Equivalently we could require $\left\langle\left(T_{t}-S_{t}\right) \omega, \tau\right\rangle \leqq K t\langle\omega, \tau\rangle$ for all $\omega \geqq 0$. This means that the probability of detecting one or more particles during the time interval $[0, t)$ gets small linearly in $t$ and uniformly in $\omega$. Although this restriction is often too strong for photon counting experiments, it is important in the fermion case and may also have applications to classical structures. As Davies has proven for CP's of this kind, $S_{t}$ is a $C_{0}$-semigroup and $\left\|\mathscr{E}_{t}\left(A_{n}^{t}\right)\right\| \leqq \frac{K^{n} t^{n}}{n !}$, i.e. the counting probability is dominated by a Poisson distribution. In the sequel let $\mathscr{E}_{t}$ be a CP with measurable space $(X, \Sigma)$. For $E \in \Sigma$ we define

$$
J_{t}(E):=\frac{1}{t} \mathscr{E}_{t}(E \times[0, t)) .
$$

The generators of the semigroups $S_{t}$ and $T_{t}$ are $W$ resp. $Z$. For shortness we write $\overline{D\left(W^{*}\right)}\|\cdot\|=: \mathscr{V}^{\odot} \subseteq \mathscr{V}^{*}$. As we want to focus mainly on the unbounded case we will prove a slightly more general statement in the subsequent lemma than is necessary for the proof of Theorem 4.2.

4.1. Lemma. Let $\mathscr{E}_{t}$ be a $C P$ with measurable space $(X, \Sigma)$ and $S_{t}$ a $C_{0}$-semigroup. Let $D\left(J^{\odot}\right)=D_{+}\left(J^{\odot}\right)-D_{+}\left(J^{\odot}\right)$ be a dense subspace of $\mathscr{V}$ invariant under $S_{t}$ and $T_{t}$ such that

$$
\left\|\left(T_{t}-S_{t}\right) S_{s} \omega\right\| \leqq K(\omega) t
$$

for $\omega \in D\left(J^{\odot}\right)$ and $s \geqq 0$. For each $\omega \in D\left(J^{\odot}\right)$ let $g_{\omega}: \mathbb{R}_{+} \rightarrow \mathbb{R}$ be a continuous function with $g_{\omega}(0)=0$ such that

$$
\left\|J_{t}(E) S_{s} \omega-J_{t}(E) S_{s^{\prime}} \omega\right\| \leqq g_{\omega}\left(\left|s-s^{\prime}\right|\right) .
$$

Then we have for $\omega \in D\left(J^{\odot}\right)$,

$$
\lim _{u \rightarrow 0} \int_{0}^{t} S_{t-s} J_{u}(E) S_{s} \omega d s=\mathscr{E}_{t}(E \times[0, t)) \omega
$$

in norm. Moreover for all $x \in \mathscr{V}^{\odot}$ and $\omega \in D\left(J^{\odot}\right)$ the limit $\lim _{t \rightarrow 0}\left\langle J_{t}(E) \omega, x\right\rangle$ exists.

Proof. It is an easy consequence of 2.1(ii) that for all $E \in \Sigma$ and $t>s$,

$$
S_{t-s} \mathscr{E}_{u}(E \times[0, u)) S_{s}=\mathscr{E}_{t+u}(E \times[0, s+u))-\mathscr{E}_{t+u}(E \times[0, s)) .
$$

Thus with a substitution $s \rightarrow s+u$ one calculates for all $\omega \in \mathscr{V}$,

$$
\int_{0}^{t} S_{t-s} \mathscr{E}_{u}(E \times[0, u)) S_{s} \omega d s=\int_{t}^{t+u} \mathscr{E}_{t+u}(E \times[0, s)) \omega d s-\int_{0}^{u} \mathscr{E}_{t+u}(E \times[0, s)) \omega d s
$$

Dividing this equation by $u$ and taking the limit $u \rightarrow 0$ we get (13). The hereby required continuity conditions can easily be obtained from (11) and strong continuity 
of $S_{t}$. Now (11) and the Alaoglu and Tychonov Theorems imply that there is a function $\Psi_{E, \omega}: \mathbb{R}_{+} \rightarrow \mathscr{V}^{* *}$ and a net $t_{\alpha} \rightarrow 0$ such that $\left\langle J_{t_{\alpha}}(E) S_{S} \omega, y\right\rangle \rightarrow\left\langle\Psi_{E, \omega}(s), y\right\rangle$ for all $y \in \mathscr{V}^{*}$. Using (12) we conclude $\left|\left\langle\Psi_{E, \omega}(s)-\Psi_{E, \omega}\left(s^{\prime}\right), y\right\rangle\right| \leqq\|y\| g_{\omega}\left(\left|s-s^{\prime}\right|\right)$. In particular $s \rightarrow \Psi_{E, \omega}(s)$ is norm continuous. For shortness let $f_{\alpha}(s):=J_{t_{\alpha}}(E) S_{s} \omega$. Now let $\varepsilon>0, \omega \in \mathscr{V} \backslash\{0\}, x \in \mathscr{V} \odot \backslash\{0\}$ and fix $t>0$. Since $S_{t}^{*}$ is a $C_{0^{-}}$ semigroup on $\mathscr{V}^{\odot}$ [6] and $g_{\omega}$ is continuous, there is a $\delta>0$ such that for $0<s<\delta:\left\|S_{s}^{*} x-x\right\|<\frac{\varepsilon}{8 K(\omega) t}$ and $g_{\omega}(s)<\frac{\varepsilon}{8\|x\| t}$. Choose a fixed $n>\frac{t}{\delta}$. Then there is an $\alpha_{0}$ such that for $\alpha \geqq \alpha_{0}$ and $k=0, \ldots, n$ we have

$$
\|\left\langle f_{\alpha}\left(\frac{k t}{n}\right)-\Psi_{E, \omega}\left(\frac{k t}{n}\right), S_{\frac{n-k-1}{n} t}^{*} x\right\rangle \mid<\frac{\varepsilon}{4 t} .
$$

Thus for $\alpha \geqq \alpha_{0}$,

$$
\begin{aligned}
\mid \int_{0}^{t}\langle & \left.S_{t-s} J_{t_{\alpha}}(E) S_{s} \omega, x\right\rangle d s-\int_{0}^{t}\left\langle\Psi_{E, \omega}(s), S_{t-s}^{*} x\right\rangle d s \mid \\
\leqq & \int_{0}^{t}\left|\left\langle f_{\alpha}(s)-\Psi_{E, \omega}(s), S_{t-s}^{*} x\right\rangle\right| d s \\
= & \sum_{k=0}^{n-1} \int_{k t / n}^{(k+1) t / n}\left|\left\langle f_{\alpha}(s)-\Psi_{E, \omega}(s), S_{t-s}^{*} x\right\rangle\right| d s \\
= & \sum_{k=0}^{n-1} \int_{0}^{t / n}\left|\left\langle f_{\alpha}\left(s+\frac{k t}{n}\right)-\Psi_{E, \omega}\left(s+\frac{k t}{n}\right), S_{\frac{n-k-1}{n} t}^{*} S_{\frac{t}{n}-s}^{*} x\right\rangle\right| d s \\
\leqq & \sum_{k=0}^{n-1} \int_{0}^{t / n}\left|\left\langle f_{\alpha}\left(s+\frac{k t}{n}\right)-\Psi_{E, \omega}\left(s+\frac{k t}{n}\right), S_{\frac{n-k-1}{n} t}^{*}\left(S_{\frac{t}{n}-s}^{*} x-x\right)\right\rangle\right| d s \\
& +\sum_{k=0}^{n-1} \int_{0}^{t / n}\left|\left\langle f_{\alpha}\left(s+\frac{k t}{n}\right)-f_{\alpha}\left(\frac{k t}{n}\right), S_{\frac{n-k-1}{n} t}^{*} x\right\rangle\right| d s \\
& +\sum_{k=0}^{n-1} \int_{0}^{t / n}\left|\left\langle f_{\alpha}\left(\frac{k t}{n}\right)-\Psi_{E, \omega}\left(\frac{k t}{n}\right), S_{\frac{n-k-1}{n} t}^{*} x\right\rangle\right| d s \\
& +\sum_{k=0}^{n-1} \int_{0}^{t / n}\left\{\left\|S_{\frac{t}{n}-s}^{*} x-x\right\| 2 K(\omega)+g_{\omega}(s)\|x\|+\frac{\varepsilon}{4 t}+g_{\omega}(s)\right\}\|x\| d s \\
& +\sum_{k=0}^{n-1} \int_{0}^{t / n}\left|\left\langle\Psi_{E, \omega}\left(\frac{k t}{n}\right)-\Psi_{E, \omega}\left(s+\frac{k t}{n}\right), S_{\frac{n-k-1}{n} t}^{*} x\right\rangle\right| d s
\end{aligned}
$$

It follows that

$$
\begin{gathered}
\left\langle\mathscr{E}_{t}(E \times[0, t)) \omega, x\right\rangle=\lim _{u \rightarrow 0} \int_{0}^{t}\left\langle S_{t-s} J_{u}(E) S_{s} \omega, x\right\rangle d s \\
=\lim _{\alpha} \int_{0}^{t}\left\langle J_{t_{\alpha}}(E) S_{s} \omega, S_{t-s}^{*} x\right\rangle d s=\int_{0}^{t}\left\langle\Psi_{E, \omega}(s), S_{t-s}^{*} x\right\rangle d s
\end{gathered}
$$


independent of the net $t_{x}$. Dividing this equation by $t$ and using the continuity of $s \rightarrow \Psi_{E, \omega}(s)$ and $s \rightarrow S_{t-s}^{*} x$ we conclude that $\lim _{t \rightarrow 0} \frac{1}{t}\left\langle\mathscr{E}_{t}(E \times[0, t)) \omega, x\right\rangle=$ $\left\langle\Psi_{E, \omega}(0), x\right\rangle$.

4.2. Theorem. Let $\mathscr{E}_{t}$ be a $C P$ with bounded $I R$ and measurable space $(X, \Sigma)$. We have $D\left(W^{*}\right)=D\left(Z^{*}\right)$ and for $\omega \in \mathscr{V}$ and $x \in \mathscr{V}^{\odot}$ the equation $\lim _{t \rightarrow 0}\left\langle J_{t}(E) \omega, x\right\rangle$ $=\langle\omega, \Phi(E) x\rangle$ defines a unique measure $\Phi: \Sigma \rightarrow \mathscr{B}\left(\mathscr{V}^{\odot}, \mathscr{V}^{*}\right)$ such that

$$
\left\langle\mathscr{E}_{t}(E \times[0, t)) \omega, x\right\rangle=\int_{s}^{t}\left\langle S_{s} \omega, \Phi(E) S_{t-s}^{*} x\right\rangle d s .
$$

The $\sigma$-additivity of $\Phi$ refers to the $\sigma\left(\mathscr{V}^{\odot}, \mathscr{V}\right)$-topology. Moreover we have $\tau \in$ $D\left(W^{*}\right)$ with $W^{*} \tau=-\Phi(X) \tau$ and on $D\left(W^{*}\right)$ we have

$$
Z^{*}=W^{*}+\Phi(X) \text {. }
$$

The CP $\mathscr{E}_{t}$ is uniquely determined by $\Phi$ and $S_{t}$.

Proof. We use the above lemma. Taking $D\left(J^{\odot}\right)=\mathscr{V}$ and using the bounded IR we conclude that $\left\|\left(T_{t}-S_{t}\right) S_{s} \omega\right\| \leqq K t$ and $\left\|J_{t}(E)\left(S_{s}-S_{s^{\prime}}\right) \omega\right\| \leqq K\left\|S_{\left|s-s^{\prime}\right|} \omega-\omega\right\|$ for all $\omega \in \mathscr{V}$. Thus we can apply Lemma 4.1 and define $\Phi(E)$ by $\lim _{t \rightarrow 0}\left\langle J_{t}(E) \omega, x\right\rangle=$ $\langle\omega, \Phi(E) x\rangle$ for $\omega \in \mathscr{V}$ and $x \in \mathscr{V} \odot$. We thus can apply the Lebesgue Theorem in (13) and get (17). By the Vitali-Hahn-Saks Theorem $E \rightarrow\langle\omega, \Phi(E) x\rangle$ is $\sigma$ additive. Now $\left\|T_{t}^{*}-S_{t}^{*}\right\| \leqq K t$ implies $D\left(Z^{*}\right)=D\left(W^{*}\right)$ (use [3, Th. 3.1.23]), and as $T_{t}^{*} \tau=\tau$ we have $Z^{*} \tau=0$ and $\tau \in D\left(W^{*}\right)$. Moreover, $\frac{1}{t}\left\|\mathscr{E}_{t}\left(\bigcup_{n \geqq 2} A_{n}^{t}\right)\right\| \rightarrow 0$ for $t \rightarrow 0$ implies $\lim _{t \rightarrow 0} \frac{1}{t}\left\|\left(T_{t}-S_{t}-\mathscr{E}_{t}(X \times[0, t))\right) \omega\right\|=0$ for all $\omega \in \mathscr{V}$, and therefore $Z^{*}=W^{*}+\Phi(X)$ on $D\left(W^{*}\right)$. Finally the last statement is an easy consequence of (17) and Lemma 3.2.

We call $\Phi$ the interaction rate of the process. This name is justified by consideration of the mean number $\langle N\rangle_{t}$ of particles detected up to the time $t$ (cf. Eq. (5)): Because of the bounded interaction rate one calculates

$$
\left.\frac{d}{d t}\langle N\rangle_{t}\right|_{t=0}=\langle\omega, \Phi(X) \tau\rangle
$$

Before giving the converse of the above result, we state the following lemma.

4.3. Lemma. Let $S_{t}, T_{t}$ be $C_{0}$-semigroups with $0<S_{t} \leqq T_{t}$ and $T_{t}^{*} \tau=\tau$, and denote $A_{u}:=\frac{1}{u}\left(T_{u}-S_{u}\right)$. Let $D_{0} \subseteq \mathscr{V}^{*}$ be invariant under $S_{t}^{*}$ and $T_{t}^{*},\left(D_{0}\right)_{+} w^{*}-$ dense in $\mathscr{V}_{+}^{*}$ and containing $\tau$. For each $t$ let $R_{t}$ be a POVM on $[0, t)$ such that

$$
\left\langle R_{t}(E) \omega, x\right\rangle=\lim _{u \rightarrow 0}\left\langle\int_{E} S_{t-s} A_{u} S_{s} \omega d s, x\right\rangle
$$

for $x \in D_{0}$ and $\omega \in \mathscr{V}$. Then $R_{t}$ satisfies condition (ii) in Theorem 3.3, and for each $l \in \mathbb{N}$ there is a POVM $\mathscr{F}_{t}^{l}$ on $C_{l}^{t}$ with $\mathscr{F}_{t}^{0}\left(\left\{z_{t}\right\}\right)=S_{t}, \mathscr{F}_{t}^{l}=R_{t}$ and

$$
\sum_{l=2}^{k} \mathscr{F}_{t}^{l}\left(C_{l}^{t}\right) \leqq T_{t}-S_{t}-R_{t}([0, t))
$$


for all $k \geqq 2$. Moreover for measurable sets $A \subseteq C_{l}^{t}$ and $B \subseteq C_{m}^{s}$ we have

$$
\mathscr{F}_{t}^{l}(A) \mathscr{\mathscr { F }}_{s}^{m}(B)=\mathscr{F}_{t+s}^{l+m}(\lambda(A \times B)) .
$$

Proof. For general semigroups $S_{t}, T_{t}$ the following identity is valid:

$$
\int_{0}^{1} S_{t-s} A_{u} T_{s} \omega d s=\frac{1}{u} \int_{t-u}^{t} S_{t-s} T_{u+s} \omega d s-\frac{1}{u} \int_{-u}^{0} S_{t-s} T_{u+s} \omega d s .
$$

By the above condition, if $\omega=\omega_{1}-\omega_{2}$ with $\omega_{1}, \omega_{2} \in \mathscr{K}$, then $\left\|T_{t} \omega\right\| \leqq\left\|T_{t} \omega_{1}\right\|+$ $\left\|T_{t} \omega_{2}\right\|=\left\|\omega_{1}\right\|+\left\|\omega_{2}\right\|$, and hence the infimum of the last term yields $\left\|T_{t} \omega\right\| \leqq$ $\|\omega\|$ so that $T_{t}$ and $S_{t}$ are contractive. Thus $\left\|\int_{0}^{t} S_{t-s} A_{u} T_{s} \omega d s\right\| \leqq 2\|\omega\|$ and

$$
\|\cdot\| \lim _{u \rightarrow 0} \int_{0}^{t} S_{t-s} A_{u} T_{s} \omega d s=T_{t} \omega-S_{t} \omega
$$

for all $\omega \in \mathscr{V}$. A careful but straightforward iteration of (23) using (19) implies

$$
\begin{gathered}
\left\langle\left(T_{t}-S_{t}\right) \omega, x\right\rangle=\left\langle R_{t}([0, t)) \omega, x\right\rangle+\lim _{u_{k} \rightarrow 0} \cdots \lim _{u_{1} \rightarrow 0} \\
\left\{\sum_{l=2}^{k-1} \int_{C_{l}^{t}}\left\langle S_{t-t_{l}} A_{u_{k}} \cdots A_{u_{k-l+1}} S_{t_{1}} \omega, x\right\rangle d t_{1} \cdots d t_{l}\right. \\
\left.\quad+\int_{C_{k}^{t}}\left\langle S_{t-t_{k}} A_{u_{k}} \cdots A_{u_{1}} T_{t_{1}} \omega, x\right\rangle d t_{1} \cdots d t_{k}\right\}
\end{gathered}
$$

for $x \in D_{0}$ and $k \geqq 2$. It is a direct consequence of (19) that $R_{t}$ satisfies condition (ii) in Theorem 3.3 for $X=\{1\}$, thus we can apply Lemma 3.4. Let $\mu_{n}^{l}$ be the sequence of POVM's on $C_{l}^{t}$ constructed there, then using (19), (8) and the Lebesgue Theorem (recall that from the above $\left\|\int_{E} S_{t-s} A_{u} S_{s} \omega d s\right\| \leqq 2\|\omega\|$ ), one calculates for standard sets $A \subseteq C_{l}^{t}$,

$$
\left\langle\mu_{n}^{l}(A) \omega, x\right\rangle=\lim _{u_{l} \rightarrow 0} \cdots \lim _{u_{1} \rightarrow 0} \int_{A}\left\langle S_{t-t_{l}} A_{u_{l}} \cdots A_{u_{1}} S_{t_{1}} \omega, x\right\rangle d t_{1} \cdots d t_{l}
$$

for sufficiently large $n$ and $x \in D_{0}$. Thus if $A_{2} \subseteq C_{2}^{t}, \ldots, A_{l} \subseteq C_{k}^{t}$ are standard sets, $\omega \in \mathscr{V}_{+}$and $x \in\left(D_{0}\right)_{+}$, we have

$$
\begin{aligned}
\sum_{l=2}^{k}\left\langle\mu_{n}^{l}\left(A_{l}\right) \omega, x\right\rangle & \leqq \liminf _{u_{k} \rightarrow 0} \cdots \liminf _{u_{l} \rightarrow 0} \sum_{l=2}^{k} \int_{C_{l}^{t}}\left\langle S_{t-t_{l}} A_{u_{k}} \cdots A_{u_{k-1+1}} S_{t_{1}} \omega, x\right\rangle d t_{1} \cdots d t_{l} \\
& \leqq\left\langle\left[T_{t}-S_{t}-R_{t}([0, t))\right] \omega, x\right\rangle
\end{aligned}
$$

where we use (24) and $T_{t} \geqq S_{t}$. But this implies $\sum_{l=2}^{k} \mu_{n}^{l}\left(C_{l}^{t}\right) \leqq T_{t}-S_{t}-R_{t}([0, t))$ $\leqq T_{t}$, thus $\| \mu_{n}^{l}\left(C_{l}^{t} \| \leqq 1\right.$ and therefore the limit

$$
\|\cdot\| \lim _{n \rightarrow \infty} \mu_{n}^{l}(E) \omega=: \mathscr{F}_{t}^{l}(E) \omega
$$

exists for all $E$ because the sequence $\mu_{n}^{l}(E)$ is monotone. By the Vitali-Hahn-Saks Theorem $\mathscr{F}_{t}^{l}$ is a POVM. Now Eq. (20) follows from (25), and (21) can easily be 
verified for standard sets (and therefore for general measurable sets) using (8) and (26).

4.4. Theorem. Let $0 \leqq S_{t} \leqq T_{t}$ be $C_{0}$-semigroups with generators $W$ resp. $Z$, and let $(X, \Sigma)$ be a standard Borel space. Define $\mathscr{V} \odot:=\overline{D\left(W^{*}\right)}\|\cdot\|$. Let $\Phi: \Sigma \rightarrow$ $\mathscr{B}_{+}\left(\mathscr{V}^{\odot}, \mathscr{V}^{*}\right)$ be a $\sigma$-additive map (in the $\sigma\left(\mathscr{V}^{\odot}, \mathscr{V}\right)$-topology) such that $W^{*} \tau=$ $-\Phi(X) \tau$ and $\left(W^{*}+\Phi(X), D\left(W^{*}\right)\right)=\left(Z^{*}, D\left(Z^{*}\right)\right)$. Moreover for each $E \in \Sigma$ and $\omega \in \mathscr{V}_{+}$let the formula

$$
\left\langle G_{t}(E \times[0, t)) \omega, x\right\rangle:=\int_{0}^{t}\left\langle S_{s} \omega, \Phi(E) S_{t-s}^{*} x\right\rangle d s, \quad x \in \mathscr{V}^{\odot}
$$

define a state $G_{t}(E \times[0, t)) \omega \in \mathscr{V}_{+}$. Then there is a unique $C P \mathscr{E}_{t}$ with measurable space $X$ such that $\mathscr{E}_{t}\left(\left\{z_{t}\right\}\right)=S_{t}$ and $\mathscr{E}_{t}(E \times[0, t)) \omega=G_{t}(E \times[0, t)) \omega$. The IR of $\mathscr{E}_{t}$ is bounded.

Proof. Define for fixed $t>0, s \in[0, t)$ and $F \subseteq X \times[0, t): F_{s}:=\{y \mid(y, s) \in F\}$. Then one can verify as in usual measure theory that for $x \in \mathscr{V} \odot$,

$$
\left\langle G_{t}(F) \omega, x\right\rangle:=\int_{0}^{t}\left\langle S_{s} \omega, \Phi\left(F_{s}\right) S_{t-s}^{*} x\right\rangle d s
$$

is well defined and $\sigma$-additive in $F$ : We can assume $\omega \geqq 0, x \geqq 0$. The set of all $F \subseteq X \times[0, t)$, where the integral on the right-hand side is well defined forms a Dynkin system containing the sets $E \times[a, b)$ for $E \in \Sigma, 0 \leqq a<b \leqq t$, thus is the whole $\sigma$-algebra. By the $\sigma$-additivity of $\Phi$ and the monotone convergence Theorem it follows that $F \rightarrow\left\langle G_{t}(F) \omega, x\right\rangle$ is a measure on $X \times[0, t)$. By definition $G_{t}(F) \omega$ is a linear functional on $\mathscr{V} \odot$. We now want to identify it with an element of $\mathscr{V}$. Thus fix $\omega \in \mathscr{V}_{+}$. Using (27) and the monotone completeness of $\mathscr{V}$ we conclude that the set of all $F \subseteq X \times[0, t)$ with $G_{t}(F) \omega \in \mathscr{V}$ forms the above Dynkin system, and therefore $F \rightarrow G_{t}(F)$ is a POVM on $X \times[0, t)$. For $x \in D\left(W^{*}\right)$ we have

$$
\begin{aligned}
\left\|A_{u}^{*} x\right\| & \leqq \frac{1}{u}\left\|\left(S_{u}^{*}-\mathbb{1}\right) x\right\|+\frac{1}{u}\left\|\left(T_{u}^{*}-\mathbb{1}\right) x\right\| \\
& \leqq\left\|W^{*} x\right\|+\left\|Z^{*} x\right\| \leqq\|\phi(X) x\|+2\left\|W^{*} x\right\|
\end{aligned}
$$

and $\lim _{u \rightarrow 0}\left\langle S_{t-s} A_{u} S_{s} \omega, x\right\rangle=\left\langle S_{s} \omega, \Phi(X) S_{t-s}^{*} x\right\rangle$. Defining $R_{t}(E):=G_{t}(X \times E)$ for measurable $E \subseteq[0, t)$ we can use the Lebesgue Theorem to get (19) for $x \in D\left(W^{*}\right)$. Now we can apply the above lemma and define

$$
\widetilde{\mathscr{E}}_{t}(B) \omega:=\sum_{l=0}^{\infty} \mathscr{F}_{t}^{l}\left(B \cap C_{l}^{t}\right) \omega
$$

for measurable $B \subseteq\{1\}_{t}$, using the POVM's $\mathscr{F}_{t}$ constructed there. The sum is converging by (20) and this inequality also implies $0 \leqq U_{t}:=\widetilde{\mathscr{E}}_{t}\left(\{1\}_{t}\right) \leqq T_{t} \cdot \widetilde{\mathscr{E}}_{t}$ therefore is a POVM on $\{1\}_{t}$. Equation (21) implies that $\widetilde{E}_{t}$ satisfies 3.3 (ii) as it suffices to verify this equation on standard sets. In particular $U_{t}$ is a semigroup. We now want to show that $U_{t}=T_{t}$. As $\tau \in D\left(W^{*}\right)$ we have for $\omega>0$,

$$
\lim _{t \rightarrow 0} \frac{1}{t}\left\|\left(T_{t}-S_{t}-G_{t}(X \times[0, t))\right) \omega\right\|=\left\langle\omega, Z^{*} \tau-W^{*} \tau+\Phi(X) \tau\right\rangle=0,
$$


and this is even true for arbitrary $\omega \in \mathscr{V}$. Thus $\omega \in D(Z)$ iff $\|\cdot\| \lim _{t \rightarrow 0} \frac{1}{t}\left(S_{t} \omega+\right.$ $\left.G_{t}(X \times[0, t)) \omega-\omega\right)=: \widetilde{\omega}$ exists, in which case we have $\widetilde{\omega}=Z \omega$. But the above equation also implies $\lim _{t \rightarrow 0} \frac{1}{t}\left\|\widetilde{\mathscr{E}}_{t}\left(\bigcup_{n \geqq 2} C_{n}^{t}\right) \omega\right\|=0$ (cf. (20)) and so we have

$$
\frac{1}{t}\left(U_{t} \omega-\omega\right)=\frac{1}{t}\left(S_{t} \omega+G_{t}(X \times[0, t)) \omega-\omega\right)+\frac{1}{t} \widetilde{\mathscr{E}}_{t}\left(\bigcup_{n \geqq 2} C_{n}^{t}\right) \omega,
$$

which is converging iff $\omega \in D(Z)$. Now it follows that $U_{t}$ is a $C_{0}$-semigroup whose generator coincides on $D(Z)$ with $Z$, thus $U_{t}=T_{t}$. By the above $\widetilde{\mathscr{E}}_{t}$ is a one point CP. We can use (27) to verify that $G_{t}$ satisfies all the conditions of Theorem 3.3 and thus defines a refinement $\mathscr{E}_{t}$ of $\widetilde{\mathscr{E}}_{t}$. In particular we have proved the existence of $\mathscr{E}_{t}$. Uniqueness again follows by Lemma 3.2 .

A combination of Theorems 4.4 and 4.2 gives a complete classification of CP's with bounded IR. This result is achieved in spite of the fact that the IR $\Phi$ in general is not $w^{*}$-continuous. In the latter case all the assumptions about $G_{t}$ are automatically satisfied, and the existence of the semigroup $T_{t}$ is immediate from perturbation theory. Thus we are interested in conditions that guarantee the existence of a predual operator $\Phi_{*}$. Recall that the Farvard class $F(W)$ of a contraction semigroup $S_{t}$ with generator $W$ is the set of all $\omega$ the orbits $\left\{S_{t} \omega \mid t \geqq 0\right\}$ of which are Lipschitz continuous [5]. For $\omega \in D(W)$ we have:

$$
\left\|S_{t} S_{s} \omega-S_{S} \omega\right\|=\left\|\int_{0}^{t} S_{r} W S_{s} \omega d r\right\| \leqq t\|W \omega\|,
$$

thus $D(W) \subseteq F(W)$. It holds always that $F(W) \subseteq D(W)$ [9] for reflexive Banach spaces, so we call $S_{t}$ "of type $R$ " iff $D(W)=F(W)$. If $J$ is a bounded operator and $Z=W+J$ generates a contraction semigroup $T_{t}$, then $\omega \in F(Z)$ implies

$$
\left\|S_{t} \omega-\omega\right\| \leqq\left\|T_{t}-S_{t}\right\|\|\omega\|+\left\|T_{t} \omega-\omega\right\| \leqq\|J\|\|\omega\| t+\left\|T_{t} \omega-\omega\right\|,
$$

thus $\omega \in F(W)=D(W)=D(Z)$ and $T_{t}$ is of type R. IF $V_{t}$ is a contraction semigroup with generator $U$ and $\left\|S_{t}-V_{t}\right\| \leqq K t$, then $\omega \in D(U)$ implies

$$
\left\|S_{t} \omega-\omega\right\| \leqq\left\|S_{t}-V_{t}\right\|\|\omega\|+\left\|V_{t} \omega-\omega\right\| \leqq(K\|\omega\|+\|U \omega\|) t
$$

and we conclude $\omega \in F(W)=D(W)$. Thus $P:=U-W$ is a well defined operator on $D(U)$ with $\|P\| \leqq K$ and $V_{t}$ is a (bounded) perturbation of $S_{t}$, hence of type $\mathrm{R}$. In the sequel we will prove that using semigroups of this type implies that the IR is $w^{*}$-continuous.

4.5. Theorem. Let $\mathscr{E}_{t}$ be a one point $C P$ with bounded IR. If $S_{t}$ is of type $R$ then $T_{t}$ is of type $R$ and there is a unique bounded operator $J: \mathscr{V} \rightarrow \mathscr{V}$ such that

$$
\mathscr{E}_{t}(E) \omega=\sum_{n=0}^{\infty} \int_{C_{n}^{t} \cap E} S_{t-t_{n}} J \cdots J S_{t_{1}} \omega d t_{1} \cdots d t_{n} .
$$

In particular the interaction rate operator $\Phi=J^{*}$ is $w^{*}$-continuous.

Proof. Defining $J=Z-W$ all except (28) is clear from the above. As both sides of (28) are $\sigma$-additive, it is sufficient to check this equation on standard sets. But using (17) and the $w^{*}$-continuity of $J^{*}$ we conclude $\mathscr{E}_{t}([0, t)) \omega=\int_{0}^{t} S_{t-s} J S_{s} \omega d s$, 
where the integral converges in norm. Now (28) is obvious for standard sets by use of some easy substitutions.

In order to show that there are nontrivial cases of semigroups of type $\mathrm{R}$, we consider the state space $\mathscr{V}$ of traditional quantum mechanics. We will often use the following result of Davies $[11,8]$.

4.6. Lemma. Let $\mathscr{H}$ be a separable Hilbert space and $\left(\varrho_{n}\right)_{n \in \mathbb{N}}$ be a sequence of positive operators in $\mathscr{V}=T_{s a}(\mathscr{H})$ which converges in the weak operator topology to $\varrho \in \mathscr{B}_{+}(\mathscr{H})$. If there is a constant $K>0$ such that $\operatorname{tr}\left(\varrho_{n}\right) \leqq K$ for all $n$, then $\varrho \in \mathscr{V}$ and $\operatorname{tr}(\varrho) \leqq \liminf _{n \rightarrow \infty} \operatorname{tr}\left(\varrho_{n}\right)$. Moreover $\left\|\varrho-\varrho_{n}\right\| \rightarrow 0$ iff $\lim _{n \rightarrow \infty} \operatorname{tr}\left(\varrho_{n}\right)=$ $\operatorname{tr}(\varrho)$.

Davies showed that, as $S_{t}$ should leave the set of pure states invariant, one has to choose $S_{t}=\operatorname{Ad} B_{t}$, i.e. $S_{t} \rho=B_{t} \rho B_{t}^{*}$ for physically interesting cases, with a contraction semigroup $B_{t}$ on $\mathscr{H}$ [11]. Now let $W$ be the generator of $S_{t}$ and $\varrho \in F(W)$. As the unit ball of $\mathscr{B}(\mathscr{H})$ is compact and metrizable in the weak operator topology, there is a sequence $t_{n} \rightarrow 0$ such that $\omega_{n}:=\frac{1}{t_{n}}\left(S_{t_{n}} \varrho-\varrho\right),\left(\omega_{n}\right)_{+}$and $\left(\omega_{n}\right)_{-}$ are converging. Applying Lemma 4.6 and [8, Lemma 5.3.1] it follows $\varrho \in D(W)$, hence $S_{t}$ is of type R. Now the result of Davies [8, Theorem 5.3.5] can be extended to semigroups of type $\mathrm{R}$.

4.7. Theorem. Let $\mathscr{H}, \mathscr{V}$ be as above, $(X, \Sigma)$ a measurable space and $\mathscr{E}_{t}$ a $C P$ with bounded interaction rate such that $S_{t}$ is of type $R$. Then the IR $\Phi$ (cf. 4.2) is $w^{*}$ continuous, i.e. there is a POVM $J: \Sigma \rightarrow \mathscr{B}_{+}(\mathscr{V}), E \rightarrow J(E)$ with $\Phi(E)=$ $\left.J^{*}(E)\right|_{\mathscr{}} \odot$ for all $E \in \Sigma$.

Proof. As above we get a sequence $t_{n} \rightarrow 0$ such that for fixed $E \in \Sigma$ and $\varrho \in \mathscr{V}_{+}$

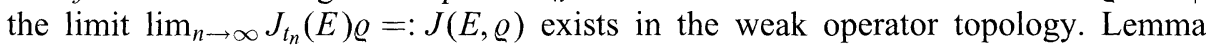
4.6 implies that $J(E, \varrho) \in \mathscr{V}_{+}$and by 4.5 for $E=X$ even $\|\cdot\|_{\operatorname{tr}} \lim _{t \rightarrow 0} J_{t}(X) \varrho=J \varrho$ exists. One can show that even $\|\cdot\|_{\mathbf{t r}} \lim _{n \rightarrow \infty} J_{t_{n}}(E) \varrho=J(E, \varrho)$. The proof of this fact is the same as the corresponding proof for pure CP's [8], we thus omit it. However, in the unbounded case we formulate explicitly similar arguments to prove Corollary 5.4. Now Theorem 4.2 shows that for $x \in D\left(W^{*}\right)$ we have: $\langle J(E, \varrho), x\rangle=$ $\langle\varrho, \Phi(E) x\rangle$, thus the limit $J(E, \varrho)$ is independent of the chosen sequence $t_{n} \rightarrow 0$. Therefore one can define $J(E) \varrho:=\lim _{n \rightarrow \infty} J_{1 / n}(E) \varrho$ and $\varrho \rightarrow J(E) \varrho$ is bounded positive operator. Obviously $E \rightarrow J(E)$ is a POVM and satisfies $J(E)^{*} x=\Phi(E) x$ for $x \in \mathscr{V}^{\odot}$.

\section{CP's with Unbounded Interaction Rate}

For physical applications the operator $J$ discussed in the previous section cannot be expected to be bounded. The usual choice would be $J \varrho=a(f) \varrho a^{*}(f)[12,13]$ with the creation and annihilation operators $a^{*}(f), a(f)$ for instance on Fock space [7]. Obviously $J$ is not bounded but well defined on a dense subspace. Thus we introduce the following definition.

5.1. Definition. $A C P \mathscr{E}_{t}$ on $\mathscr{V}$ is said to have an (unbounded) interaction rate, if there is a dense space $D^{\prime} \subseteq \mathscr{V}$ such that for each $\omega \in D^{\prime}$ there is a $K(\omega)>0$ with

$$
\left\|\left(T_{t}-S_{t}\right) \omega\right\| \leqq K(\omega) t \quad \text { for all } t>0
$$


As $S_{t}$ is contractive and strongly continuous on the dense space $D^{\prime}$, it follows that $S_{t}$ is a $C_{0}$-semigroup whose generator we denote by $W$. There are two instructive conditions implying that the IR is bounded. First if $\tau \in D\left(W^{*}\right)$ one easily verifies that $\left\|T_{t}-S_{t}\right\| \leqq\left\|W^{*} \tau\right\|$. Second if (29) holds for all $\omega \in \mathscr{V}$, the uniform boundedness principle implies that the IR is bounded. Both are conditions that complicate the handling with CP's of this type. As "physically relevant" states will satisfy (29) (cf. (6)) we will require $D(Z) \cup D(W) \subseteq D^{\prime}$. In the bounded case we have seen that it is reasonable to concentrate on semigroups $S_{t}$ (and thus $T_{t}$ ) of type R. But requiring $S_{t}$ and $T_{t}$ of type $\mathrm{R}$ here implies for $\omega \in D(W)$,

$$
\left\|T_{t} \omega-\omega\right\| \leqq\left\|\left(T_{t}-S_{t}\right) \omega\right\|+\left\|S_{t} \omega-\omega\right\| \leqq K(\omega) t+\|W \omega\| t,
$$

thus $\omega \in D(Z)$ and vice versa. In the sequel we therefore require $D(Z)=D(W)$. We call an (unbounded) operator $J: D \subseteq \mathscr{V} \rightarrow \mathscr{V}$ positive if $D_{+}=\{\omega \in D \mid \omega \geqq 0\}$ is dense in $\mathscr{V}_{+}$and $J \omega \geqq 0$ for $\omega \in D_{+}$.

5.2. Proposition. Let $\mathscr{E}_{t}$ be a $C P$ with $D(Z)=D(W)$. Then the operator $(J, D(J))$ $:=(Z-W, D(W))$ is well defined and positive. $J, W$ and $Z$ are relatively bounded to $W$ and $Z$ and there are constants $a, b>0$ such that for all $\omega \in D(W)$ and $u, s>0$,

$$
\left\|\frac{1}{u}\left(T_{u}-S_{u}\right) S_{S} \omega\right\| \leqq a\|\omega\|+b\|W \omega\| .
$$

Proof. As $S_{t}$ is positive, $D_{+}(W)$ is dense in $\mathscr{V}_{+}$. Thus $J \omega=\lim _{u \rightarrow 0} \frac{1}{u}\left(T_{u}-S_{u}\right) \omega$ implies that $J$ is positive. If $\{(\omega, W \omega) \mid \omega \in D(W)\}=: G_{W}$ is the graph of $W$, then considering the operator $\widetilde{Z}(\omega, W \omega):=Z \omega$ and using the closed graph theorem, we conclude that $Z$ (and therefore $J=Z-W$ ) is relatively bounded to $W$. In the same way it follows that $W$ is relatively bounded to $Z$. Finally (30) is a consequence of the semigroup identity $T_{u} \omega-\omega=\int_{0}^{u} T_{r} Z \omega d r$ for $\omega \in D(Z)$ and the above.

We now want to show that in the unbounded case there is also an operator characterizing the interaction rate. It is necessary to have such a result in order to justify the usual way of constructing models - by taking an unbounded operator $J$ - as the only possibility to do this. Recall that $\mathscr{V}^{\odot}:={\overline{D\left(W^{*}\right)}}^{\|\cdot\|}$ is an ordered Banach space $\left(S_{t}\right.$ is positive). For shortness let $J_{t}(E):=\frac{1}{t} \mathscr{E}_{t}(E \times[0, t))$ for $E \in \Sigma$ and $D\left(J^{\odot}\right):=D_{+}(W)-D_{+}(W)$. Obviously $D\left(J^{\odot}\right)$ is dense in $\mathscr{V}$.

5.3. Theorem. Let $\mathscr{E}_{t}$ be an $C P$ with $D(Z)=D(W)$. For each $E \in \Sigma$ and $\omega \in D\left(J^{\odot}\right)$ there is a unique operator $J^{\odot}(E): D\left(J^{\odot}\right) \rightarrow \mathscr{V}^{\odot *}$ satisfying $\lim _{t \rightarrow 0}\left\langle J_{t}(E) \omega, x\right\rangle=\left\langle J^{\odot}(E) \omega, x\right\rangle$ for all $x \in \mathscr{V} \odot$. Therefore $E \rightarrow\left\langle J^{\odot}(E) \omega, x\right\rangle$ is $\sigma$-additive and we have

$$
\left\langle\mathscr{E}_{t}(E \times[0, t)) \omega, x\right\rangle=\int_{0}^{t}\left\langle J^{\odot}(E) S_{s} \omega, S_{t-s}^{*} x\right\rangle d s
$$

for all $x \in \mathscr{V} \odot, \omega \in \mathscr{V}$ and $E \in \Sigma$. Moreover for $E=X$ and for all $\omega \in D(W)$ we have

$$
\mathscr{E}_{t}(X \times[0, t)) \omega=\int_{0}^{t} S_{t-s} J S_{s} \omega d s,
$$

where the integral converges in norm. Thus we can identify $J^{\odot}(X)$ with $J$. 
Proof. For shortness let $A_{u}:=\frac{1}{u}\left(T_{u}-S_{u}\right)$. Looking at Proposition 5.2 it remains to show the validity of Eq. (12) in order to apply Lemma 4.1. Now for $\omega \in D(W)$ let $W \omega=(W \omega)_{+}-(W \omega)_{-}$be a fixed decomposition of $W \omega$ in positive parts and $\varphi:=(W \omega)_{+}+(W \omega)_{-}$. Then for $s, s^{\prime}>0$,

$$
\begin{aligned}
\left\|J_{t}(E) S_{s} \omega-J_{t}(E) S_{s^{\prime}} \omega\right\| & =\left\|J_{t}(E) \int_{s}^{s^{\prime}} S_{u} W \omega d u\right\| \\
& \leqq\left\|J_{t}(E) \int_{s}^{s^{\prime}} S_{u}(W \omega)_{+} d u\right\|+\left\|J_{t}(E) \int_{s}^{s^{\prime}} S_{u}(W \omega)_{-} d u\right\| \\
& =\left\langle J_{t}(E) \int_{s}^{s^{\prime}} S_{u} \varphi d u, \tau\right\rangle \leqq \frac{1}{t}\left\langle\left(T_{t}-S_{t}\right) \int_{s}^{s^{\prime}} S_{u} \varphi d u, \tau\right\rangle \\
& \leqq a\left\|\int_{s} S_{u} \varphi d u\right\|+b\left\|W \int_{0}^{s^{\prime}-s \mid} S_{u} \varphi d u\right\| \\
& \leqq a\left|s-s^{\prime}\right|\|\varphi\|+b\left\|\left(S_{\left|s-s^{\prime}\right|}-\mathbb{1}\right) \varphi\right\| .
\end{aligned}
$$

By Lemma 4.1 we now can define $\left\langle J^{\odot}(E) \omega, x\right\rangle:=\lim _{t \rightarrow 0}\left\langle J_{t}(E) \omega, x\right\rangle$ and the VitaliHahn-Saks Theorem implies that $E \rightarrow\left\langle J^{\odot}(E) \omega, x\right\rangle$ is $\sigma$-additive. Thus (31) is a direct consequence of Eq. (16), using $\left\langle\Psi_{E, \omega}(s), S_{t-s}^{*} x\right\rangle=\left\langle J^{\odot}(E) S_{s} \omega, S_{t-s}^{*} x\right\rangle$. For $u, t>0$ and measurable $E \subseteq[0, t)$ define $R_{t}^{u}(E) \omega:=\int_{E} S_{t-s} A_{u} S_{s} \omega d s$. Using (22) and $S_{s} \leqq T_{s}$ we see that $\left\|R_{t}^{u}(E)\right\| \leqq 2$. By the Alaoglu and Tychonov Theorems there is a net $u_{\alpha} \rightarrow 0$ and a bounded operator $R_{t}(E): \mathscr{V} \rightarrow \mathscr{V}^{* *}$ such that

$$
\left\langle R_{t}^{u_{\alpha}}(E) \omega, x\right\rangle \rightarrow\left\langle R_{t}(E) \omega, x\right\rangle
$$

for all $\omega \in \mathscr{V}$ and $x \in \mathscr{V}^{*}$. But Eq. (30) and the Lebesgue Theorem imply that

$$
R_{t}(E) \omega=\int_{E} S_{t-s} J S_{s} \omega d s
$$

for $\omega \in D(W)$, where the integral converges in norm. Thus the limit in (34) is independent of the net $u_{x}$, and $E \rightarrow R_{t}(E)$ is a POVM on $[0, t)$ satisfying (19) (with $D_{0}=\mathscr{V}^{*}$ ). For the remaining proof of (32) we can assume $X=\{1\}$. Then

$$
\left.\mathscr{E}_{t}([0, t)) \omega=\lim _{u \rightarrow 0} \int_{0}^{t} S_{t-s} J_{u}(\{1\})\right) S_{s} \omega d s \leqq \lim _{u \rightarrow 0} \int_{0}^{t} S_{t-s} A_{u} S_{s} \omega d s=R_{t}([0, t)) \omega,
$$

where the limit exists in the weak topology. We can apply Lemma 4.3 to $R_{t}$, and get POVM's $\mathscr{F}_{t}^{l}$ on $C_{l}^{t}$. Then (21) and (36) imply that $\mathscr{E}_{t}\left(C_{n}^{t}\right) \leqq \mathscr{F}_{n}^{t}\left(C_{n}^{t}\right)$. Thus we have for $\omega>0$,

$$
T_{t} \omega=\sum_{n=0}^{\infty} \mathscr{E}_{t}\left(C_{n}^{t}\right) \omega \leqq \sum_{n=0}^{\infty} \widetilde{F}_{n}^{t}\left(C_{n}^{t}\right) \omega \leqq T_{t} \omega
$$

where the last inequality follows from $(20)$. Therefore we conclude $\mathscr{E}_{t}([0, t))=$ $R_{t}([0, t))$. 
The above theorem shows that there is an operator valued measure characterizing the interaction rate. The result lacks one fact: the images of the operator $J^{\odot}(E)$ are in $\mathscr{V}^{\odot *}$ rather than in $\mathscr{V}$. We note however that this problem does not arise, if we focus our attention on one point CP's (cf. Eq. (32)). There is a better result obtainable for special state spaces.

5.4. Corollary. Let $\mathscr{H}$ be a separable Hilbert space, $\mathscr{V}:=\mathscr{T}_{s a}(\mathscr{H}), \mathscr{E}_{t}$ a $C P$ on $\mathscr{V}$ with $D(Z)=D(W)$. Then for each $E \in \Sigma$ there is a positive operator $J(E)$ : $D\left(J^{\odot}\right) \rightarrow \mathscr{V}$ such that

$$
\mathscr{E}_{t}(E \times[0, t)) \omega=\int_{0}^{t} S_{t-s} J(E) S_{s} \omega d s
$$

for all $\omega \in D\left(J^{\odot}\right)$, where the integral converges in norm. Furthermore $E \rightarrow J(E) \omega$ is $\sigma$-additive and

$$
J(E) \omega=\|\cdot\| \lim _{t \rightarrow 0} J_{t}(E) \omega .
$$

Proof. Let $\omega \in D_{+}(W)$. As the unit ball of $\mathscr{B}(\mathscr{H})$ is compact and metrizable in the weak operator topology, the inequality $\left\|J_{t}(E) \omega\right\| \leqq\left\|A_{t} \omega\right\| \leqq a\|\omega\|+b\|W \omega\|$ implies the existence of a sequence $t_{n} \rightarrow 0$ such that $J_{t_{n}}(E) \omega \rightarrow J(E, \omega) \in \mathscr{B}(\mathscr{H})$. By Lemma 4.6 we have $J(E, \omega) \in \mathscr{V}_{+}$and Theorem 5.3 implies that for $E=X$ even $\|\cdot\| \lim _{t \rightarrow 0} J_{t}(X) \omega=J \omega$. Thus for $E^{c}=X \backslash E$,

$$
\lim _{n \rightarrow \infty} J_{t_{n}}\left(E^{c}\right) \varrho=\lim _{n \rightarrow \infty}\left(J_{t_{n}}(X) \varrho-J_{t_{n}}(E) \varrho\right)=J \varrho-J(E, \varrho)=: J\left(E^{c}, \varrho\right)
$$

exists in the weak operator topology and the limit is in $\mathscr{V}$. Now applying Lemma 4.6 to a subsequence $u_{k}=t_{n_{k}}$ of $t_{n}$ shows

$$
\begin{aligned}
\operatorname{tr}(J \varrho) & =\liminf _{k \rightarrow \infty} \operatorname{tr}\left(J_{u_{k}}(X) \varrho\right) \\
& \geqq \liminf _{k \rightarrow \infty} \operatorname{tr}\left(J_{u_{k}}(E) \varrho\right)+\liminf _{k \rightarrow \infty} \operatorname{tr}\left(J_{u_{k}}\left(E^{c}\right) \varrho\right) \\
& \geqq \operatorname{tr}(J(E, \varrho))+\operatorname{tr}\left(J\left(E^{c}, \varrho\right)\right)=\operatorname{tr}(J \varrho),
\end{aligned}
$$

and therefore $\liminf _{k \rightarrow \infty} \operatorname{tr}\left(J_{t_{n_{k}}}(E) \varrho\right)=\operatorname{tr}(J(E, \varrho))$ for any subsequence of $t_{n}$. We conclude $\lim _{n \rightarrow \infty} \operatorname{tr}\left(J_{t_{n}}(E) \varrho\right)=\operatorname{tr}(J(E, \varrho))$ and by 4.6 we have

$$
\|\cdot\|_{\operatorname{tr}} \lim _{n \rightarrow \infty} J_{t_{n}}(E) \varrho=J(E, \varrho) .
$$

By Theorem 5.3 we have $\left\langle J^{\odot}(E) \omega, x\right\rangle=\langle J(E, \omega), x\rangle$ for $x \in \mathscr{V} \odot$, hence the limit $J(E, \omega)$ is independent of the chosen sequence $t_{n} \rightarrow 0$. Thus we can define the operator $J(E)$ by (38). Using (33) and an $\varepsilon / 3$ argument, we see that $s \rightarrow J(E) S_{s} \omega$ is continuous in norm. Therefore the integral in (37) is well defined, and (37) follows from (31).

Now we come to the commutation relations of a process.

5.5. Theorem. Let $\mathscr{E}_{t}$ and $\mathscr{V}$ as in 5.4, $D_{1}:=\left\{\omega \in D_{+}(W)-D_{+}(W) \mid W \omega \in\right.$ $\left.D_{+}(W)-D_{+}(W)\right\}$. Then $D_{1}$ is a core for $W$. Moreover, for $\omega \in D_{1}$ and $E \in \Sigma$ we have $\mathscr{E}_{t}(E \times[0, t)) \omega \in D(W)$ with

$$
W \mathscr{E}_{t}(E \times[0, t)) \omega=S_{t} J(E) \omega-J(E) S_{t} \omega+\int_{0}^{t} S_{t-s} J(E) S_{s} W \omega d s .
$$


Thus on $D_{1}$ the CP fulfills the following commutation relations:

$$
\left[W, \mathscr{\delta}_{t}(E \times[0, t))\right]=\left[S_{t}, J(E)\right] .
$$

Proof. The span of all $\omega_{t}=\int_{0}^{t} S_{s}(\omega) d s$, where $t>0$ and $(1) \in D_{+}(W)$ is obviously dense in $y$, contained in $D_{1}$ and invariant under $S_{t}$, thus $D_{1}$ is a core for $W$. For (1) $\in D_{1}$, Eq. (37) implies

$$
\begin{aligned}
& \left(S_{r}-\mathbb{1}\right) \mathscr{E}_{t}(E \times[0, t)) \omega=\int_{t}^{t+r} S_{s} J(E) S_{t+r-s}(\omega) d s-\int_{0}^{r} S_{s} J(E) S_{t-s} \omega d s \\
& \quad+\int_{r}^{t} S_{s} J(E)\left(S_{t+r-s}(\omega)-S_{t-s} \omega\right) d s .
\end{aligned}
$$

Dividing this equation by $r$ and taking the limit $r \rightarrow 0$ leads to (39). Equation (40) then follows from (37).

These relations are also valid in the bounded case, where the right-hand side of (40) is always bounded! After having studied the properties of CP's, we want to construct them starting out from the generators $J$ and $W$. We first concentrate on one point processes. As in semigroup theory there is no general answer to this perturbation problem, but the subsequent theorem shows that it is enough to solve the semigroup problems.

5.6. Theorem. Let $S_{t}$ be a positive semigroup with generator $(W, D(W))$, and $(J, D(J))$ a positive operator such that $W+J$ is closable on $D=D(W) \cap D(J)$, and the closure $Z$ is the generator of a positive semigroup $T_{t}$ satisfying $T_{t}^{*} \tau=\tau$ for all $t$. Let $D_{+}$be dense in $\mathscr{t}_{+}$, invariant under $S_{t}$ and $T_{t}$, and for $\omega \in D_{+}$let $s \rightarrow J T_{s} \omega$ and $s \rightarrow J S_{s}(1)$ be continuous functions. Then there is a unique one point $C P \mathscr{E}_{t}$ with $\mathscr{E}_{t}\left(\left\{z_{t}\right\}\right)=S_{t}$ and $\mathscr{E}_{t}([0, t)) \omega=\int_{0}^{t} S_{t-s} J S_{s} \omega d s$ for $\omega \in D$. Moreover $\mathscr{E}_{t}\left(X_{t}\right)=T_{t}$.

Proof. Positivity of $T_{t}$ and $T_{t}^{*} \tau=\tau$ imply $\left\|T_{t}\right\| \leqq 1$. Let $A_{u}:=\frac{1}{u}\left(T_{u}-S_{u}\right)$. The function $s \rightarrow W T_{s}(1)=(Z-J) T_{s} \omega$ is continuous for $\omega \in D_{+}$and therefore bounded on $[0, t]$. Thus there is a constant $M(\omega)>0$ such that for all $u>0$ and $s \in$ $[0, t]$,

$$
\begin{aligned}
\left\|A_{u} T_{s} \omega\right\| & \leqq\left\|\frac{1}{u}\left(T_{u}-\mathbb{1}\right) T_{s} \omega\right\|+\left\|\frac{1}{u}\left(S_{u}-\mathbb{1}\right) T_{s}(\omega)\right\| \\
& =\left\|\frac{1}{u} \int_{0}^{u} T_{r} Z T_{s} \omega d r\right\|+\left\|\frac{1}{u} \int_{0}^{u} S_{1} W T_{s}(1) d r\right\| \leqq M(\omega) .
\end{aligned}
$$

Now we can apply the Lebesgue Theorem in Eq. (23) and get

$$
T_{t}(\omega)-S_{t}(\omega)=\int_{0}^{t} S_{t-s} J T_{s}(1) d s .
$$

We conclude $T_{t} \geqq S_{t}$ and thus (22) implies $\left\|\int_{E} S_{t-s} A_{u} S_{s}(\omega) d s\right\| \leqq 2\|\omega\|$ for all $\omega \in \mathcal{H}^{\circ}$. As above (cf. (34) and (35)) we conclude that there is a bounded positive operator $R(E) \in \mathscr{B}_{+}(\mathscr{\mathscr { Y }})$ such that for $\omega \in D_{+}$we have $R(E) \omega=\int_{E} S_{t-s} J S_{s} \omega d s$. Obviously $E \rightarrow R(E)$ is a POVM on $[0, t)$ and we can apply Lemma 4.3 . Let $\widetilde{\mathscr{H}}_{t}^{n}$ be the POVM on $C_{n}^{t}$ constructed there. We define

$$
\mathscr{E}_{t}(E) \omega:=\sum_{n=0}^{\infty} \widetilde{\mathscr{F}}_{t}^{n}\left(E \cap C_{n}^{t}\right) \omega
$$


By (20) this is a well defined POVM on $\{1\}_{t}$ with $\mathscr{E}_{t}\left(\{1\}_{t}\right)=: U_{t} \leqq T_{t}$. As it suffices to verify 2.1 (ii) for standard sets, the Markov properties follow from (21). In particular $U_{t}$ is a semigroup. It remains to show $U_{t}=T_{t}$. Now for $\omega \in D_{+}$we have $\lim _{t \rightarrow 0} \frac{1}{t} \mathscr{F}_{t}^{1}([0, t)) \omega=\lim _{t \rightarrow 0} \frac{1}{t} \int_{0}^{t} S_{t-s} J S_{s} \omega d s=J \omega$ and $\lim _{t \rightarrow 0} \frac{1}{t}\left(\mathscr{F}_{t}^{0}\left(\left\{z_{t}\right\}\right) \omega-\right.$ $\omega)=W \omega$. Therefore we have

$$
\lim _{t \rightarrow 0} \mathscr{E}_{t}\left(\bigcup_{n \geqq 2} C_{n}^{t}\right) \omega \leqq \lim _{t \rightarrow 0}\left\{\frac{1}{t}\left(T_{t}-S_{t}\right) \omega-\frac{1}{t} R_{t}([0, t)) \omega\right\}=J \omega-J \omega=0,
$$

which implies

$$
\lim _{t \rightarrow 0} \frac{1}{t}\left(U_{t} \omega-\omega\right)=W \omega+J \omega=Z \omega
$$

In particular we have $\left\|U_{t} \omega-\omega\right\| \rightarrow 0$ for $t \rightarrow 0$, and since $\left\|U_{t}\right\| \leqq 1$ and $D_{+}$is dense in $\mathscr{V}_{+}, U_{t}$ is a $C_{0}$-semigroup whose generator coincides on the core $D_{+}-$ $D_{+}$with $Z$, hence $U_{t}=T_{t}$.

We note that if $J$ is relatively bounded to $W$ with relative bound smaller than 1 , most of the conditions of the above theorem are automatically fulfilled. Now we come to the refinements of such processes. If $W$ and $J$ determine the process $\mathscr{E}_{t}$, then looking at 5.3 and 5.4 suggests that a refinement can be constructed by giving a "measure" $E \rightarrow J(E)$ with $J(X)=J$.

5.7. Definition. Let $(J, D(J))$ be a positive operator and $(X, \Sigma)$ a measurable space. For each $E \in \Sigma$ let $J(E): D_{+}(J)-D_{+}(J) \rightarrow \mathscr{V}$ be a positive operator and $J(X)=J$. If for each $\omega \in D_{+}(J)$ the map $E \rightarrow J(E) \omega$ is $\sigma$-additive, then $E \rightarrow$ $J(E)$ is called a decomposition of $J$ on $(X, \Sigma)$.

In most cases a decomposition of $J$ will define a refinement of the corresponding $\mathrm{CP}$. The subsequent theorem will give sufficient conditions.

5.8. Theorem. Let $(J, D(J))$ be a positive operator such that for all $\omega \in D_{+}(J)-$ $D_{+}(J)$ there are $\omega_{1}, \omega_{2} \in D_{+}(J)$ with $\omega=\omega_{1}-\omega_{2}$ and

$$
\|\omega\| \leqq \lambda\left(\left\|\omega_{1}\right\|+\left\|\omega_{2}\right\|\right)
$$

for some $\lambda>0$. Let $\tilde{\mathscr{E}}_{t}$ be a one point counting process such that $S_{t} D_{+}(J) \subseteq$ $D_{+}(J)$ and $\mathscr{E}_{t}([0, t)) \omega=\int_{0}^{t} S_{t-s} J S_{S} \omega d s$ for all $\omega \in D_{+}(J)$. Let $(X, \Sigma)$ be a standard Borel space and $E \rightarrow J(E)$ a decomposition of $J$ on $(X, \Sigma)$ such that for $\omega \in D_{+}(J)$ the functions $s \rightarrow J(E) S_{s} \omega$ are continuous in norm. Then there is a unique $C P \mathscr{E}_{t}$ with measurable space $(X, \Sigma)$ such that for all $\omega \in D_{+}(J)$,

$$
\mathscr{E}_{t}(E \times[0, t)) \omega=\int_{0}^{t} S_{t-s} J(E) S_{s} \omega d s
$$

Proof. For measurable $F \subseteq X \times[0, t)$ let $F_{s}:=\{y \in X \mid(y, s) \in F\}$. Then define for $x \in \mathscr{V}^{*}$ and $\omega \in D_{+}(J)-D_{+}(J)$,

$$
\left\langle G_{t}(F) \omega, x\right\rangle=\int_{0}^{t}\left\langle S_{t-s} J\left(F_{s}\right) S_{s} \omega, x\right\rangle d s .
$$


Obviously the left-hand side is well defined and linear in $x$ and $\omega$. Moreover, for $\omega=\omega_{1}-\omega_{2}$ as in (41) we have

$$
\begin{aligned}
\left|\left\langle G_{t}(F) \omega, x\right\rangle\right| & \leqq \int_{0}^{t}\left\|S_{t-s} J\left(F_{s}\right) S_{s}\left(\omega_{1}+\omega_{2}\right)\right\|\|x\| d s \\
& \leqq \int_{0}^{t}\left\langle S_{t-s} J(X) S_{s}\left(\omega_{1}+\omega_{2}\right), \tau\right\rangle\|x\| d s \\
& =\left\langle\mathscr{E}_{t}([0, t))\left(\omega_{1}+\omega_{2}\right), \tau\right\rangle\|x\| \leqq \lambda\|\omega\|\|x\| .
\end{aligned}
$$

Thus each $G_{t}(F)$ can be uniquely extended to a positive bounded operator $\mathscr{V} \rightarrow$ $\mathscr{V}^{* *}$. But $G_{t}(F) \omega \in \mathscr{V}$ for standard sets $F$ and $\omega \in D(J)$ (and therefore all $\omega \in \mathscr{V}$ ) because of the norm continuity of the functions $s \rightarrow J(E) S_{s} \omega$. Now use the $\sigma$ additivity of $G_{t}$ - which is obvious from (43)-and the monotone completeness of $\mathscr{V}$ in order to verify that for fixed $\omega \in \mathscr{V}_{+}$the set of all $F$ such that $G_{t}(F) \omega \in \mathscr{V}$ forms a Dynkin system, containing the standard sets, hence $G_{t}(F) \in \mathscr{B}_{+}(\mathscr{V})$ for all $F$. Using (43) it is easy to verify that one can apply Theorem 3.3 to get the desired result.

We want to make some remarks about the conditions of the above theorem. If $J$ is relatively bounded to $W$ and $D_{+}(J)=D_{+}(W)$, a similar estimation as in (33) shows that all the functions $s \rightarrow J(E) S_{s} \omega$ are continuous for any decomposition of $J$. So the main problem in applications of the theorem is to check (41). Consider a pure CP (i.e. $S_{t}$ is a pure operation) on $\mathscr{V}=\mathscr{T}_{s a}(\mathscr{H})$. We have $S_{t}=\operatorname{Ad} B_{t}$, and if $Y$ is the generator of $B_{t}$, then $D:=\mathbf{L H}\left\{|\psi\rangle\langle\varphi|| \psi, \varphi \in D(Y)\} \cap \mathscr{T}_{s a}(\mathscr{H})\right.$ is obviously dense in $\mathscr{V}$, invariant under $S_{t}$ and a subset of $D(W)$. Moreover it is a simple application of the spectral theorem for finite dimensional Hilbert spaces that if $\rho \in D$ then also the parts $\rho_{+}$and $\rho_{-}$of the Jordan decomposition of $\rho$ are contained in $D$. Thus $D$ satisfies (41) with $\lambda=1$. A combination of these arguments together with 5.3 and 5.4 gives the following corollary:

5.9. Corollary. Let $\mathscr{H}$ be a separable Hilbert space, $\mathscr{V}=\mathscr{T}_{\text {sa }}(\mathscr{H})$ and $\widetilde{\mathscr{E}}_{t}$ a pure one point $C P$ with $D(W)=D(Z)$. Let $J:=Z-W$ and $(X, \Sigma)$ a standard Borel space. Then there is a one-to-one correspondence between the following classes:

(i) Refinements $\mathscr{E}_{t}$ of $\widetilde{\mathscr{E}}_{t}$ on $(X, \Sigma)$.

(ii) Decompositions $E \rightarrow J(E)$ of $J$ on $(X, \Sigma)$.

The correspondence is given by (42) for $E \in \Sigma$ and $\omega \in D_{+}(W)$.

We finally want to give a few comments on how the above results are related to the work of Davies on unbounded interaction rates. First, we presented a systematic theory of unbounded IR's on general state spaces, while the models constructed in [11] and [12] always used the trace class operators on Fock space. Second, the IR in these models was bounded on the $n$-particle subspaces. We emphasize that Theorem 5.6 allows to take interaction rates that are unbounded on the $n$-particle subspaces even on Fock space. Finally, the general theory makes it possible to deal with representations of the CCR-algebra that are different from the Fock representation. There the state space is the predual of the represented $W^{*}$-algebra and the IR on the $n$-particle subspaces (if there are some) can be unbounded.

Acknowledgement. The author wants to thank Prof. Dr. A. Rieckers, J. Hertle, T. Gerisch, S. Zanzinger and J. Peeck for useful discussions and a critical reading of the manuscript. Further the financial support by a LGFG-grant from the University Tübingen is gratefully acknowledged. 


\section{References}

1. Barchielli, A.: Direct and heterodyne detection and other applications of quantum stochastic calculus to quantum optics. Quantum Opt. 2, 423-441 (1990)

2. Barchielli, A.: Detection Theory in Quantum Optics and Quantum Stochastic Calculus. Lecture Notes in Physics 378, Berlin, Heidelberg, New York: Springer, 1991

3. Bratteli, O., Robinson, D.W.: Operator Algebras and Quantum Statistical Mechanics I. BerlinHeidelberg-New York: Springer Verlag, 1987

4. Bratteli, O., Robinson, D.W.: Operator Algebras and Quantum Statistical Mechanics II. New York, Heidelberg, Berlin: Springer, 1981

5. Butzer, P.L., Behrens, H.: Semigroups of Operators and Approximation. Berlin, Heidelberg, New York: Springer, 1967

6. Clement, P., Diekmann, O., Gyllenberg, M., Heijmans, H.J.A.M., Thieme, H.R.: Perturbation Theory for Dual Semigroups, I. The Sun-Reflexive Case. Math. Ann. 277, 709-725 (1987)

7. Cook, J.: The mathematics of second quantization. Trans. Am. Math. Soc. 74, 222-245 (1953)

8. Davies, E.B.: Quantum Theory of Open Systems. New York, London: Academic Press, 1976

9. Davies, E.B.: One-Parameter-Semigroups. New York, London: Academic Press, 1980

10. Davies, E.B.: An Operational Approach to Quantum Probability. Commun. Math. Phys. 17, 239-260 (1970)

11. Davies, E.B.: Quantum Stochastic Processes. Commun. Math. Phys. 15, 277-304 (1969)

12. Davies, E.B.: Quantum Stochastic Processes III. Commun. Math. Phys. 22, 51-70 (1971)

13. Davies, E.B., Srinivas, M. D.: Photon Counting Probabilities in Quantum Optics. Optica Acta 28, 981-996 (1981)

14. Dunford, N., Schwartz, J. T.: Linear Operators I. New York, London: Interscience Publ., 1958

15. Edwards, C.M.: The operational approach to algebraic quantum theory I. Commun. Math. Phys. 16, 207-230 (1970)

16. Glauber, R.J.: The Quantum Theory of Optical Coherence. Phys. Rev. 130,(6) 2529-39 (1963)

17. Hanche-Olsen, H., Størmer, E.: Jordan Operator Algebras. Boston: Pitman, 1984

18. Holmes, C.A., Milburn, G.J., Walls, D.F.: Photon-number-state preparation in nondegenerate parametric amplification. Phys. Rev A 39, 5, 2493-2501 (1989)

19. Honegger, R., Rapp, A.: General Glauber Coherent States on the Weylalgebra and their Phase Integrals. Physica A 167, 945-961 (1990)

20. Honegger, R., Rieckers, A.: The General Form of non-Fock Coherent Boson States. Publ. RIMS Kyoto Univ. 26, 397-417 (1990)

21. Honegger, R., Rieckers, A.: First Order Coherent Boson States. Preprint (1991)

22. Imoto, N., Ueda, M., Ogawa, T.: Microscopic theory of the continuous measurement of photon number. Phys. Rev. A 41, 7, 4127-4130 (1990)

23. Imoto, N., Ueda, M., Ogawa, T.: Continuous quantum-nondemolition measurement of photon number. Phys. Rev. A 46, 5, 2859-2869 (1992)

24. Kelley, P.L., Kleiner, W.H.: Theory of Electromagnetic Field Measurement and Photoelectron Counting. Phys. Rev. 136, 2A, A316-A334 (1964)

25. Mandel, L., Sudarshan, E.C.G., Wolf, E.: Theory of photoelectric detection of light fluctuations. Proc. Phys. Soc. 84, 435-444 (1964)

26. Marte, M.A.M., Zoller, P.: Lasers with sub-Poissonian pump. Phys. Rev A 40, 10, 5774-5782 (1989)

27. Milburn, G.J., Walls, D.F.: State reduction in quantum-counting quantum nondemolition measurements. Phys. Rev. A 30, 1, 56-60 (1984)

28. Mollow, B.R.: Quantum Theory of Field Attenuation. Phys. Rev. 168, 5, 1896-1919 (1968)

29. Scully, M.O., Lamb, W.E.: Quantum Theory of an Optical Maser. III. Theory of Photoelectron Counting Statistics. Phys. Rev. 179, 2, 368-374 (1969)

30. Zoller, P., Marte, M., Walls, D.F.: Quantum jumps in atomic systems. Phys. Rev. A 35, 1, 198-207 (1986) 